\title{
Nonassociated and Coupled Flow Rules of Elastoplasticity for Rock-Like Materials*
}

\author{
G. MAIER + \\ T. HUECKEL $\ddagger$
}

\begin{abstract}
Incremental elastoplastic stress-strain relations are studied which allow for non association of plastic strain rates with the (smooth) current yield surface (non-normality), and/or changing elastic moduli because of plastic deformations (elastoplastic coupling). These circumstances, often accompanied by strainsoftening, are significant in various geotechnical media, particularly rocks. On the basis of a suitable description of the rate relations, various ranges of the hardening modulus are established and their distinct behavioural characteristics, such as rate response uniqueness and material stability (or lack thereof) are systematically studied and interpreted in mechanical terms. Specialization to the standard compression tests and for particular forms of constitutive laws is made. Similarities to and differencies from the traditional associated flow rules of elastoplasticity are pointed out.
\end{abstract}

\section{INTRODUCTION}

In a broad class of engineering situations caused by mining and surface earth works, the mechanical behaviour of rocks may be fairly realistically described by means of a (time-scale insensitive) elastoplastic material model $[1,2,3]$. However, a direct application of traditional elastoplasticity may be inadequate, because some phenomenological features of rocklike materials are clearly incompatible with metal plasticity and require a revision of some of its basic concepts.

The elastoplastic material characterization in the traditional elastoplastic theory, involves the following ingredients: a constant, symmetric, positive definite tensor of elastic moduli; a 'yield function' of the stress tensor, which defines (as the locus where it is negative) the current elastic domain in the space of the stress components; a plastic flow rule which relates plastic strain rates to stress rates and is assumed to be associated as specified below; a hardening rule, according to which some parameters contained in the yield function depend on some measures of the irreversible deformation process (such as volumetric plastic strains or, more generally, 'internal variables') see e.g. $[4,5]$. The flow rule is said to be 'associated' to the yield function

* This paper was presented in parts at the 18th Polish Solid Mechanics Conference, Wisła, Sept. 1976, and the 9th Int. Congress on Soil Mechanics and Foundation Engineering, Tokyo, July 1977.

$\dagger$ Department of Structural Engineering. Technical University (Politecnico) Milan, Italy.

† Department of Structural Engineering, Technical University (Politecnico) Milan, Italy. On leave of absence from Polish Academy of Sciences, Institute of Fundamental Technological Research, Warsaw, Poland. when the plastic strain rate vector, in the strain space superposed to the stress space, is directed as the outward normal to the (smooth) 'yield surface' which forms the contour of the convex elastic domain. Plastic strain increments are defined as the difference between the measurable (total) incremental strains in a yielding process and the elastic incremental strains corresponding, through the elastic law, to the same stress increments. The yield surface in an incremental yielding process, as the stress point moves, may expand giving rise to hardening behaviour, or remain unaltered (perfectly plastic behaviour). In these cases the material is regarded as 'stable' in the sense of Drucker [6].

For rock-like materials, the following new phenomenological aspects become quite significant: (a) the plastic strain rate vector has a direction, still independent from stress rates, but different from the outward normal to the yield surface in the stress point and related to the plastic dilatancy (non-normality); (b) the elastic moduli change as inelastic deformations develop (elastic-plastic coupling); (c) in an incremental yielding process, the yield surface may either locally expand (hardening behaviour), or shrink ('softening' behaviour), or remain unaltered (perfectly plastic or 'critical' states).

Sometimes in the rock mechanics literature (e.g. in [7]) the adjectives 'ductile' and 'brittle' are used for hardening and perfectly plastic behaviour and for softening behaviour, respectively, though they usually refer to the ability to sustain significant plastic deformations.

Figure 1 shows a customary plot, fitting tri-axial tests on a specimen of a rock material which can be regarded as isotropic. The dotted lines are stress paths which 


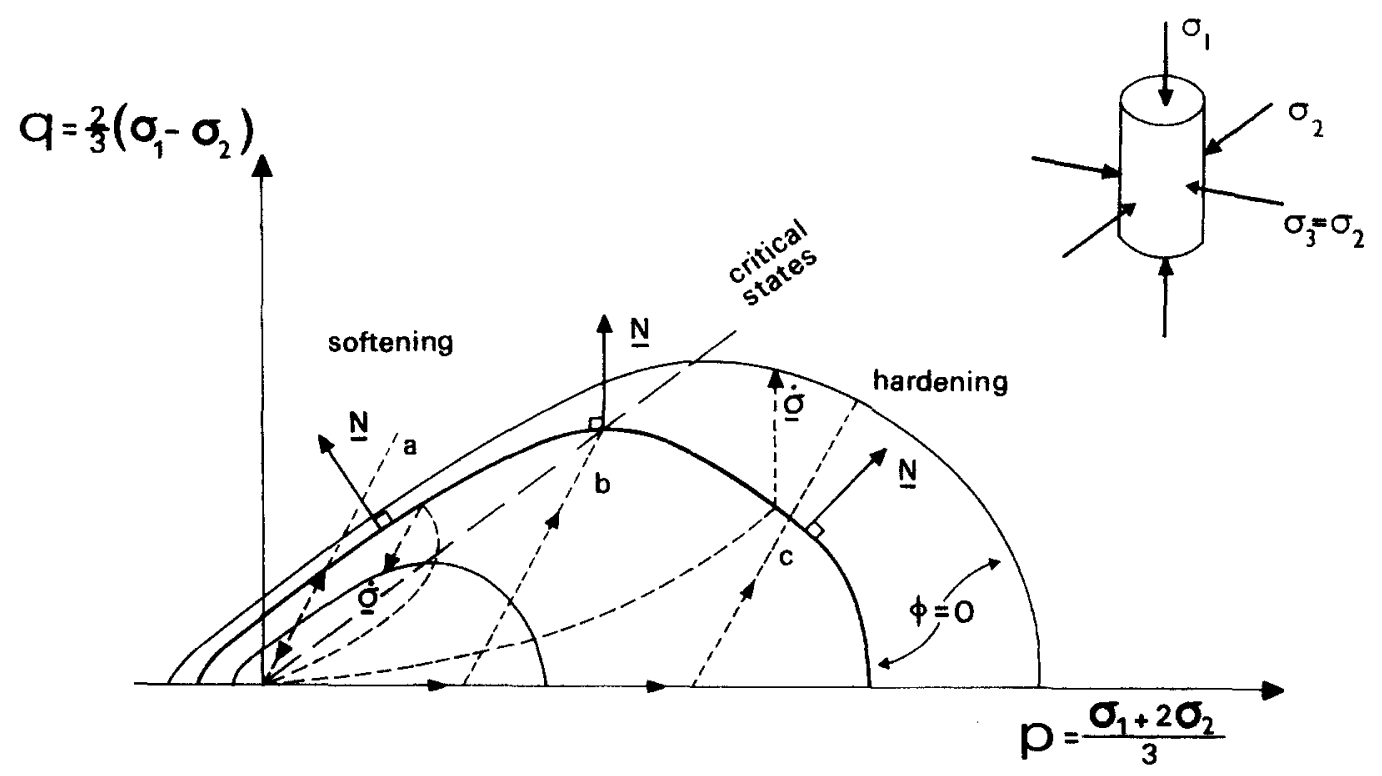

Fig. 1. A typical idealized representation of the behaviour of a rock in triaxial shear tests.

lead to incremental processes, as those which are indicated by $\dot{\sigma}$ and imply hardening and softening $[3,8]$. The shown changes of the yield surface correspond to isotropic hardening and are controlled by the volu+ metric plastic strains. The experimental plot of Fig. 2, concerning a uniaxial test on a sandstone specimen, visualizes a strong coupling effect (b), together with pronounced softening (c) $[9,10,11]$.

Softening is a peculiar, intriguing feature of inelastic behaviour of some materials. It has been studied both by allowing for strain localization and relevant discontinuities $[12,13]$, and by accommodating it in the familiar concept of material constitutive laws based on the assumption of the possibility of homogeneous strain and stress states $[14,15,16,17]$. The latter approach, which generally leads to easier tractable boundary value problems will be adopted here. Associated elastoplastic flow laws allowing for softening were studied in [18]. Non-associated incremental stress-strain laws were previously investigated, e.g. in $[19,20]$, and recently in [12]. Constitutive laws accommodating elastoplastic coupling were considered in $[21,22]$.

In this paper elastoplastic coupling and nonnormality are recognized to be amenable to the unified model of the so-called 'linear' non-associated flow rules of elastoplasticity (Section 2). These relations are systematically studied with reference to smooth yield surfaces, for all possible ranges of instantaneous behaviour.

The deviation from normality (a), the coupling phenomenon (b) and the plastic softening (c) may substantially affect the overall responses to external actions of mechanical systems, e.g. rocks undergoing excavations and surface loads. The results achieved here on

* In what follows the underlined symbols are used for vectors and matrices for geometrical visualization in figures, while components of tensors referred to the Cartesian system of reference are written in index notation. The index summation convention is adopted throughout. uniqueness of incremental response and on material stability appear to be of use for the subsequent analysis of rock masses and engineering systems exhibiting the title effects, as expounded elsewhere [23].

\section{FORMULATION OF THE RATE RELATIONS}

Let dots denote rates (understood as derivatives with respect to any monotonously increasing function $t$ of the physical time) and let $\epsilon_{i j}$ indicate the (symmetric) tensor of small strains. Strain rates $\dot{\epsilon}_{i j}$ for a yielding incremental process over $\delta t$, can be conceived as consisting of a reversible part $\dot{\epsilon}_{i j}^{R}$ and an irreversible part $\dot{\epsilon}_{i j}^{I}$. The reversible addend reads:

$$
\dot{\epsilon}_{i j}^{R}=C_{i j k l} \dot{\sigma}_{k l} \text {, }
$$

where $C_{i j k l}$ is the current elastic compliance tensor, with the usual symmetries, at the situation $\Sigma$ from which the irreversible process starts. $\Sigma$ is characterized by the (symmetric) stress tensor $\sigma_{i j}$ and the preceding plastic

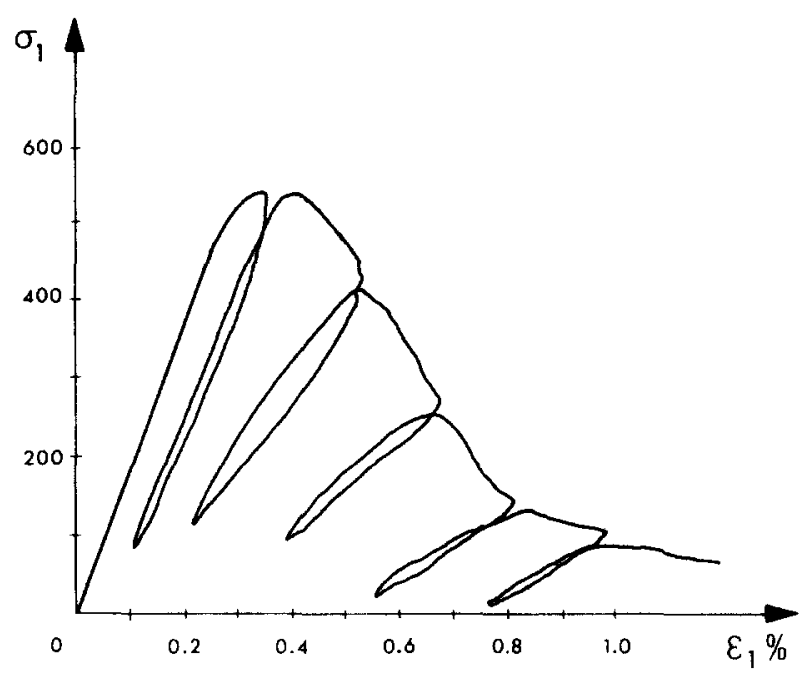

Fig. 2. Typical experimental plot from uniaxial compression of a hard rock specimen. 


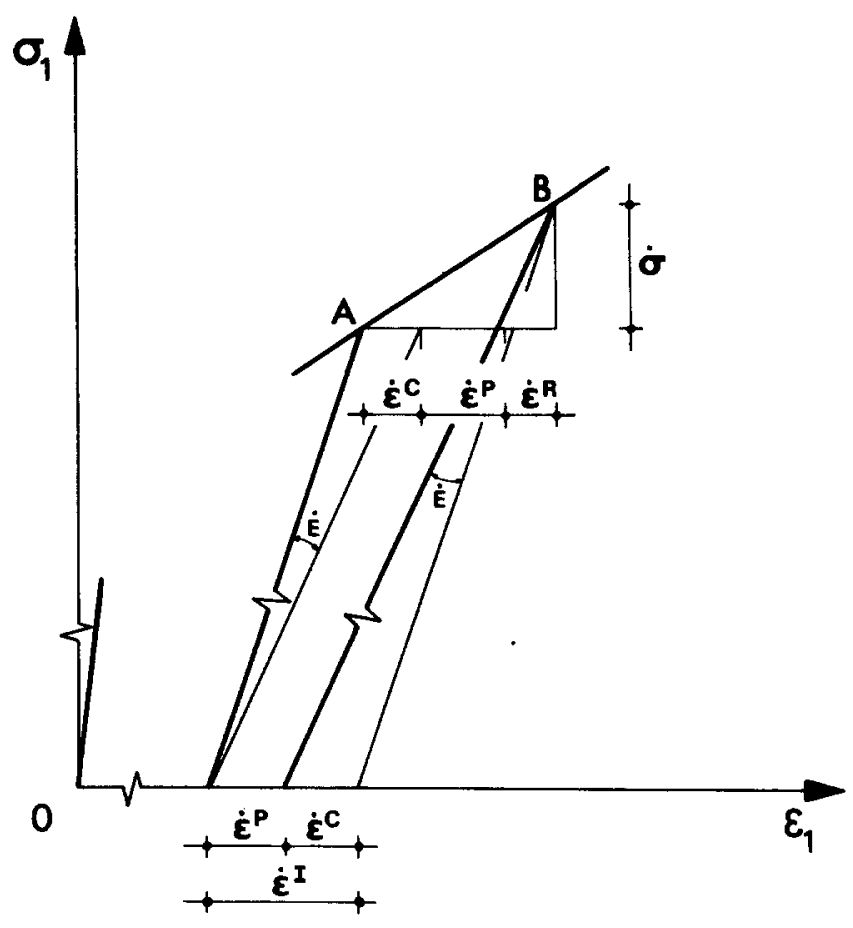

Fig. 3. Schematic idealized representation of a uniaxial incremental stress-strain relation in the presence of coupling (increments are replaced by rates for convenience of representation).

history II (i.e. some set of 'internal' or 'hidden' parameters). The tensor $C_{i j k l}$ may depend on the stress state $\sigma_{i j}$ (nonlinear elasticity) and on the plastic strains developed previously (elasto-plastic coupling).

The irreversible part $\dot{\epsilon}_{i j}^{I}$ is defined as the difference between the total strain rate $\dot{\epsilon}_{i j}$ and $\dot{\epsilon}_{i j}^{R}$ given by equation (2.1), for the same $\dot{\sigma}_{i j}$. As an alternative definition, $\dot{\epsilon}_{i j}^{I} \delta t$ represents the strain increment which remains after the yield-generating stress increment $\dot{\sigma}_{i, j} \delta t$ have been removed $\dagger$.

In turn, $\dot{\epsilon}_{i j}^{I}$ can be regarded as composed of a plastic $\dot{\epsilon}_{i j}^{p}$ and a coupled $\dot{\epsilon}_{i j}^{c}$ part (see Fig. 3). The plastic strain increments $\dot{\epsilon}_{i j}^{p} \delta t$ are singled out by removal of all stresses; the coupled strain increment defined as $\dot{\epsilon}_{i j}^{c}$ $\delta t=\left(\dot{\epsilon}_{i j}^{I}-\dot{\epsilon}_{i j}^{p}\right) \delta t$, is caused by changes in elastic moduli due to yielding

$$
\dot{\epsilon}_{i j}^{c}=\frac{\partial C_{i j k l}}{\partial \epsilon_{r s}^{p}} \dot{\epsilon}_{r s}^{p} \sigma_{k l} .
$$

The meaning of the various addends of $\dot{\epsilon}_{i j}$ is illustrated in Fig. 3 with reference to a one-dimensional situation and assuming linear elasticity. Interpreted as a vector in the $\epsilon_{i j}$ - space, the plastic strain rate vector $\dot{\epsilon}_{i j}^{p}$ is directed along the gradient vector of a (differentiable) plastic potential function $\psi\left(\sigma_{i j}, \Pi\right)$ of the stresses $\sigma_{i j}$ and of the previous plastic history $\Pi$ :

$$
\dot{\epsilon}_{i j}^{p}=\frac{\partial \psi}{\partial \sigma_{i j}} \lambda
$$

\section{$\dot{\lambda}$ being a nonnegative scalar ('plastic multiplier').}

† Note that irreversible strain increments according to the forme definition, as difference $\left(\dot{\epsilon}_{i j}-\dot{\epsilon}_{i j}^{R}\right) \delta t$, differ by second order infinitesimal from those according to the latter definition. It is also worth noting that the wording 'irreversible', 'nonrecoverable' is justified inasmuch it is referred here to incremental strains.
Let $\phi\left(\sigma_{i j}, \Pi\right)$ denote the (differentiable) yield function, such that $\phi . \leq 0$ defines the current elastic domain in the $\sigma_{i j}$ - space. If the functions $\phi$ and $\psi$ coincide, the flow rule $(2.3)$ is called associative. This restriction, which would be implied by Drucker postulate [3], is not assumed in what follows. By differentiating $\phi$, one obtains:

$$
\dot{\phi}=\frac{\partial \phi}{\partial \sigma_{i j}} \dot{\sigma}_{i j}+\frac{\partial \phi}{\partial \epsilon_{i j}^{p}} \dot{\epsilon}_{i j}^{p} .
$$

The plastic flow rules for a situation $\Sigma$ at the yield limit $(\phi=0)$ require:

$$
\dot{\lambda} \geq 0, \dot{\phi} \leq 0, \dot{\phi} \dot{\lambda}=0
$$

The third of relations (2.5) implies that plastic yielding ( $\dot{\lambda}>0$, 'loading' process) and loss of contact of the stress point with the current yield surface $(\dot{\phi}<0$, 'unloading process') are events which rule out each other.

The above tensor equations can be re-written as follows, for the sake of simplicity:

$$
\begin{gathered}
\dot{\phi}=N_{i j} \dot{\sigma}_{i j}-H \dot{\lambda} \\
\dot{\epsilon}_{i j}=C_{i j k l} \dot{\sigma}_{k l}+V_{i j} \dot{\lambda}
\end{gathered}
$$

having set:

$$
\begin{gathered}
V_{i j} \equiv \frac{\partial \psi}{\partial \sigma_{i j}}+\frac{\partial C_{i j h k}}{\partial \epsilon_{r s}^{p}} \frac{\partial \psi}{\partial \sigma_{r s}} \sigma_{h k} \\
N_{i j} \equiv \frac{\partial \phi}{\partial \sigma_{i j}}, H \equiv-\frac{\partial \phi}{\partial \epsilon_{i j}^{p}} \frac{\partial \psi}{\partial \sigma_{i j}} .
\end{gathered}
$$

Moreover, let $E_{i j k l}$ be the inverse of the elastic compliance tensor i.e. the current tangent elastic stiffness tensor; this will be assumed as always positive definite in the sense that positive definiteness is exhibited by the relevant quadratic form in the six independent components of the (symmetric) tensor $\dot{\epsilon}_{i j}$. The quantities defined by equations (2.8)-(2.9) depend on the starting situation, but not on increments. The scalar $H$ will be referred to as 'hardening modulus'. $H>0$ characterizes hardening, $H=0$ perfectly plastic, $H<0$ softening behaviour. Tensor $\dot{\epsilon}_{i j}$, making its addends explicit, can be expressed as:

$$
\dot{\epsilon}_{i j}=\dot{\epsilon}_{i j}^{R}+\dot{\epsilon}_{i j}^{I}=\dot{\epsilon}_{i j}^{R}+\dot{\epsilon}_{i j}^{p}+\dot{\epsilon}_{i j}^{c}
$$

The relation set (2.5)-(2.7) defines the dependence of the strain rates on the stress rates (direct flow rules) for a situation $\Sigma$. This dependence in the $\sigma_{i j}, \epsilon_{i j}$ spaces superposed, is schematically illustrated in Fig. 4. The inverse strain-stress rate laws will be also used in subsequent developments. From (2.6)-(2.7):

$$
\begin{gathered}
\dot{\sigma}_{i j}=E_{i j k l}\left(\dot{\epsilon}_{k l}-V_{k l} \dot{\lambda}\right) \\
\dot{\phi}=M_{i j} \dot{\epsilon}_{i j}-\left(H-H_{c}\right) \dot{\lambda}
\end{gathered}
$$

where:

$$
\begin{gathered}
M_{i j}=E_{i j k l} N_{k l} \\
H_{c}=-N_{i j} E_{i j k l} V_{k l}
\end{gathered}
$$




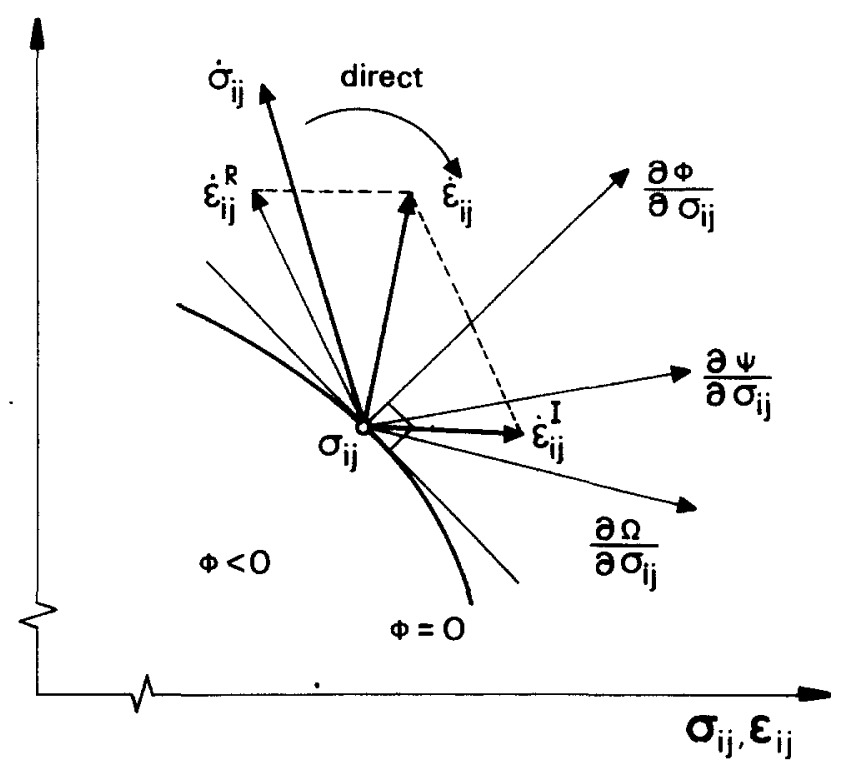

Fig. 4. Incremental elastoplastic law with deviation of the irreversible strain rate vector from the outward normal to the yield locus.

The inverse flow laws are analytically described by the relation set $(2.5),(2.11)-(2.12)$.

Tensors $\dot{\epsilon}_{i j}$ and $\dot{\sigma}_{i j}$ have six independent components. However, in the absence of significant anisotropy, the material description can be carried out in terms of principal stresses and strains. Thus, the geometric visualization in Fig. 4, 5, 7 and 11 can be conceived in terms of three-dimensional vectors $\dot{\epsilon}$ and $\dot{\sigma}$.

The above formulated rate laws are 'linear' nonassociated elastoplastic flow-rules, in the sense that all relations are linear in the rates, except (2.5). As a consequence of this so-called linearity, over the whole set of incremental 'loading' processes $(\dot{\lambda}>0, \dot{\phi}=0)$ from a given situation, the rate relations become truly linear and the direction of the irreversible strain rate vectors is fixed.

\section{UNIQUENESS AND EXISTENCE OF STRAIN (STRESS) RATE RESPONSE TO GIVEN STRESS (STRAIN) RATES}

Consider an incremental process which involves plastic yielding, i.e. $\dot{\lambda}>0, \dot{\phi}=0$, hence $\dot{\epsilon}_{i, j}^{I} \neq 0$. The direct flow laws (2.5)-(2.7), unless $H=0$, furnish:

$$
\begin{gathered}
\dot{\lambda}_{i}=\frac{1}{H} N_{i j} \dot{\sigma}_{i j} \\
\dot{\epsilon}_{i j}=A_{i j k l} \dot{\sigma}_{k l}
\end{gathered}
$$

where :

$$
A_{i j k l}=C_{i j k l}+\frac{1}{H} V_{i j} N_{k l}
$$

From the inverse flow laws (2.5), (2.11) (2.12), unless $H=H_{c}$, one obtains :

$$
\begin{aligned}
\dot{\lambda} & =\frac{1}{H-H_{c}} M_{i j} \dot{\epsilon}_{i j} \\
\dot{\sigma}_{i j} & =B_{i j k l} \dot{\epsilon}_{k l}
\end{aligned}
$$

where:

$$
\begin{aligned}
B_{i j k l} & =E_{i j k l}-\frac{1}{H-H_{c}} U_{i j} M_{k l} \\
U_{i j} & =E_{i j k l} V_{k l} .
\end{aligned}
$$

The tensors $A_{i j k l}$ and $B_{i j k l}$ are the direct (compliance) and inverse (stiffness) elastoplastic tensors. Note that $A_{i j k l} \neq A_{k l i j}$ in general, but $A_{i j k l}=A_{i j l k}$ and $A_{i j k l}$ $=A_{j i k l}$ as a consequence of the symmetry of $\dot{\sigma}_{i j}$ and $\dot{\epsilon}_{i j}$; the same holds for $B_{i j k l}$. Clearly, for $H \neq 0$ and $\neq H_{c}$, the matrices of the components of these fourthorder tensors are nonsingular and one is the inverse of the other.

For a purely elastic incremental process $(\dot{\lambda}=0$, $\dot{\phi} \leq 0$ )

$$
\dot{\epsilon}_{i j}=\dot{\epsilon}_{i j}^{R}=C_{i j k l} \dot{\sigma}_{k l}
$$

In discussing the rate relations formulated in Section 2 , a first question concerns existence of the strain rate response (of the material element free of external constraints, with homogeneous stress and strain states) for given stress rates.

An incremental stress path is 'admissible' if there is an incremental strain path corresponding to it through the direct law. By interchanging 'stress' and 'strain', a similar question arises about the inverse law. Another problem of interest in the same context concerns the multiplicity of strain (stress) rate responses for given stress (strain) rates.

Questions of these types can be straightforwardly answered on the basis of the above relations.

Consider, e.g. a stress response to $\dot{\epsilon}_{i j}$ such that $M_{i j} \dot{\epsilon}_{i j}>0$.

Assume first $H>H_{c}$. Equation (3.4) is compatible with the condition that $\dot{\lambda} \geq 0$ and defines $\dot{\lambda}$. Therefore, through equation (2.11) there exists an incremental stress response $\dot{\sigma}_{i j}$. This $\dot{\sigma}_{i j}$ is uniquely defined by equation (3.4) and (2.11), since an elastic response $(\dot{\lambda}=0)$ is ruled out for $M_{i j} \dot{\epsilon}_{i j}>0$ by equation (2.12) and by the sign requirement on $\dot{\phi}$.

Assume now $H<H_{c}$. Equation (3.4) for elastoplastic processes is in conflict with the sign constraint on $\dot{\lambda}$; equation (2.12) for elastic processes is in contradiction with $\dot{\phi} \leq 0$.

Finally, if $H=H_{c}$, equation (2.12) and $\dot{\phi} \leq 0$ again contradict $M_{i j} \dot{\epsilon}_{i j}>0$. Therefore, for $H \leq H_{c}$ any vector $\dot{\epsilon}_{i j}$ such that $M_{i j} \dot{\epsilon}_{i j}>0$ is not admissible, in the sense that there is no corresponding incremental stress response.

Conclusions of this sort are systematically presented below. 
For given $\dot{\sigma}_{\mathrm{ij}}$ existence of $\dot{\epsilon}_{\mathrm{ij}}\left(\right.$ admissibility of $\left.\dot{\sigma}_{\mathrm{ij}}\right)$, uniqueness of $\dot{\epsilon}_{\mathrm{ij}}$ :

\begin{tabular}{|c|c|c|c|c|c|c|}
\hline$N_{i j} \dot{\sigma}_{i j}>0:$ & for & $\begin{array}{ll}H>0: & \dot{\sigma}_{i j} \text { admissible, } \\
H=0: & \dot{\sigma}_{i j} \text { not admissible, } \\
H<0: & \dot{\sigma}_{i j} \text { not admissible, }\end{array}$ & & $\begin{array}{c}\lambda>0 \\
- \\
-\end{array}$ & & $\begin{array}{c}\dot{\epsilon}_{i j} \text { unique } \\
- \\
-\end{array}$ \\
\hline$N_{i j} \dot{\sigma}_{i j}=0:$ & for & $\begin{array}{ll}H>0: & \dot{\sigma}_{i j} \text { admissible } \\
H=0: & \dot{\sigma}_{i j} \text { admissible } \\
H<0: & \dot{\sigma}_{i j} \text { admissible }\end{array}$ & $\lambda>0$ & $\begin{array}{l}\lambda=0 \\
\text { or } \\
\lambda=0\end{array}$ & $\lambda=0$ & $\begin{array}{l}\dot{\epsilon}_{i j} \text { unique } \\
\dot{\epsilon}_{i j} \text { not unique (there are } \infty \dot{\epsilon}_{i j} \text { ) } \\
\dot{\epsilon}_{i j} \text { unique }\end{array}$ \\
\hline$N_{i j} \dot{\sigma}_{i j}<0:$ & for & $\begin{array}{ll}H>0: & \dot{\sigma}_{i j} \text { admissible } \\
H=0: & \dot{\sigma}_{i j} \text { admissible } \\
H<0: & \dot{\sigma}_{i j} \text { admissible }\end{array}$ & $\lambda=0$ & $\begin{array}{l}\lambda=0 \\
\lambda=0, \\
\quad \text { or }\end{array}$ & $\lambda>0$ & $\begin{array}{l}\dot{\epsilon}_{i j} \text { unique } \\
\dot{\epsilon}_{i j} \text { unique } \\
\dot{\epsilon}_{i j} \text { not unique (there are two } \dot{\epsilon}_{i j} \text { ) }\end{array}$ \\
\hline
\end{tabular}

For given $\dot{\epsilon}_{\mathrm{ij}}$ existence of $\dot{\sigma}_{\mathrm{ij}}$ (admissibility of $\dot{\epsilon}_{\mathrm{ij}}$ ), uniqueness of $\dot{\sigma}_{\mathrm{ij}}$ :

\begin{tabular}{|c|c|c|c|c|c|c|}
\hline$M_{i j} \dot{\epsilon}_{i j}>0:$ & for & $\begin{array}{l}H>H_{c}: \dot{\epsilon}_{i j} \text { admissible, } \\
H=H_{c}: \dot{\epsilon}_{i j} \text { not admissible } \\
H<H_{c}: \dot{\epsilon}_{i j} \text { not admissible }\end{array}$ & & $\begin{array}{l}\lambda>0 \\
- \\
-\end{array}$ & & $\begin{array}{c}\dot{\sigma}_{i j} \text { unique } \\
-\end{array}$ \\
\hline$M_{i j} \dot{\epsilon}_{i j}=0:$ & for & $\begin{array}{l}H>H_{c}: \dot{\epsilon}_{i j} \text { admissible, } \\
H=H_{c}: \dot{\epsilon}_{i j} \text { admissible, } \\
H<H_{c}: \dot{\epsilon}_{i j} \text { admissible, }\end{array}$ & $\lambda>0$ & $\begin{array}{l}\lambda=0 \\
\text { or } \\
\lambda=0\end{array}$ & $\lambda=0$ & $\begin{array}{l}\dot{\sigma}_{i j} \text { unique } \\
\dot{\sigma}_{i j} \text { not unique (there are } \infty \dot{\sigma}_{i j} \text { ) } \\
\dot{\sigma}_{i j} \text { unique }\end{array}$ \\
\hline$M_{i j} \dot{\epsilon}_{i j}<0:$ & for & $\begin{array}{l}H>H_{c}: \dot{\epsilon}_{i j} \text { admissible, } \\
H=H_{c}: \dot{\epsilon}_{i j} \text { admissible, } \\
H<H_{c}: \dot{\epsilon}_{i j} \text { admissible, }\end{array}$ & $\lambda=0$ & $\begin{array}{l}\lambda=0 \\
\lambda=0 \\
\quad \text { or }\end{array}$ & $\lambda>0$ & $\begin{array}{l}\dot{\sigma}_{i j} \text { unique } \\
\dot{\sigma}_{i j} \text { unique } \\
\dot{\sigma}_{i j} \text { not unique (there are two } \dot{\sigma}_{i j} \text { ) }\end{array}$ \\
\hline
\end{tabular}

The above results are illustrated in Fig. 5 and clarified by the following comments.

(i) The direct $\dot{\sigma}_{i j} \rightarrow \dot{\epsilon}_{i j}$ and inverse $\dot{\epsilon}_{i j} \rightarrow \dot{\sigma}_{i j}$ relations, if considered within suitable ranges of the hardening modulus $H$, exhibit full analogy. This is visualized in Fig. 5, where the left column (a, c, e) concerns the direct law, the right column $(b, d, f)$ the inverse one, and the correspondences by analogy are between (a) and (b); (c) and (d); (e) and (f).

(ii) Admissibility, in the previously specified sense, of all incremental stress paths holds only for $H>0$, whereas any incremental strain path is admissible for $H>H_{c}$ (Fig. 5a and b). The circumstance that for $H \leq H_{\mathrm{c}}$ a strain rat tensor for which $M_{i j} \dot{\epsilon}_{i j}>0$ is not admissible, means that, at the given situation, there is a discontinuity ('a finite jump') in the stresses, when the deformation proceeds along such path $\dot{\epsilon}_{i j}$. This circumstance will be discussed further in Section 5 .

(iii) Uniqueness of strain rate response fails to hold in the range $H \leq 0$; for softening behaviour, $H<0$, there are two and only two $\dot{\epsilon}_{i j}$ (one purely elastic and the other elastic-plastic) for any inward $\dot{\sigma}_{i j}$ (Fig. 5e). Analogously, there is no uniqueness of the stress rates in the range $H \leq H_{c}$; for $H<H_{c}$ ('subcritical' softening) there are always one elastic and one elastoplastic stress rate response for any $\dot{\epsilon}_{i j}$ such that $M_{i j} \dot{\epsilon}_{i j}<0$ (Fig. 5f).

(iv) For $H=H_{c}$ ('perfectly brittle' behaviour or 'critical' softening), there is an infinity of stress rate responses, including one purely elastic, to any $\dot{\epsilon}_{i j}$ such that $M_{i j} \dot{\epsilon}_{i j}=0$ (Fig. $5 \mathrm{~d}$ ); note the analogy to the perfectly plastic behaviour $(H=0$, Fig. $5 \mathrm{c})$. Existence and uniqueness (or lack thereof) of the rate response for various ranges of the hardening modulus $H$ are indicated in Fig. 6, where also some conclusions on mater- ial stability obtained in the subsequent Section are visualized for comparison.

\section{MATERIAL STABILITY}

Mechanical stability is usually meant as a tendency to preserve the equilibrium configuration despite disturbances; in other terms, a stable system responds to any geometric disturbance with a motion, such that suitable measures of its amplitude at any subsequent time tend to zero as measures of the disturbance tend to zero.

Stability of time-independent materials in a given state under dead loading can be connected with the non-negativeness of the second-order work density performed by an external agency over any infinitesimal path leading from the considered actual situation to any neighbouring (virtual) configuration. A sufficient condition for mechanical stability in the above sense was identified by Hill [24] as the non-negativeness of the second-order work density performed along any proportional path; in terms of rates (i.e. to within $\delta t^{2}$ ):

$$
W=\frac{1}{2} \dot{\sigma}_{i j} \dot{\epsilon}_{i j} \geq 0 \text {, for any } \dot{\epsilon}_{i j}
$$

This inequality turns out to be implied by Drucker's stability criterion 'in the small' [6].

The general concept of mechanical stability mentioned at the beginning of this Section, does not necessarily imply the above requirement in terms of energy, as shown by counterexamples in [25].

Therefore criterion (4.1) should be regarded as sufficient, not necessary, for stability in the aforementioned sense. However, for the sake of simplicity, the criterion (4.1) will be employed as a definition of stability in what follows; in other words, the material will be regarded 

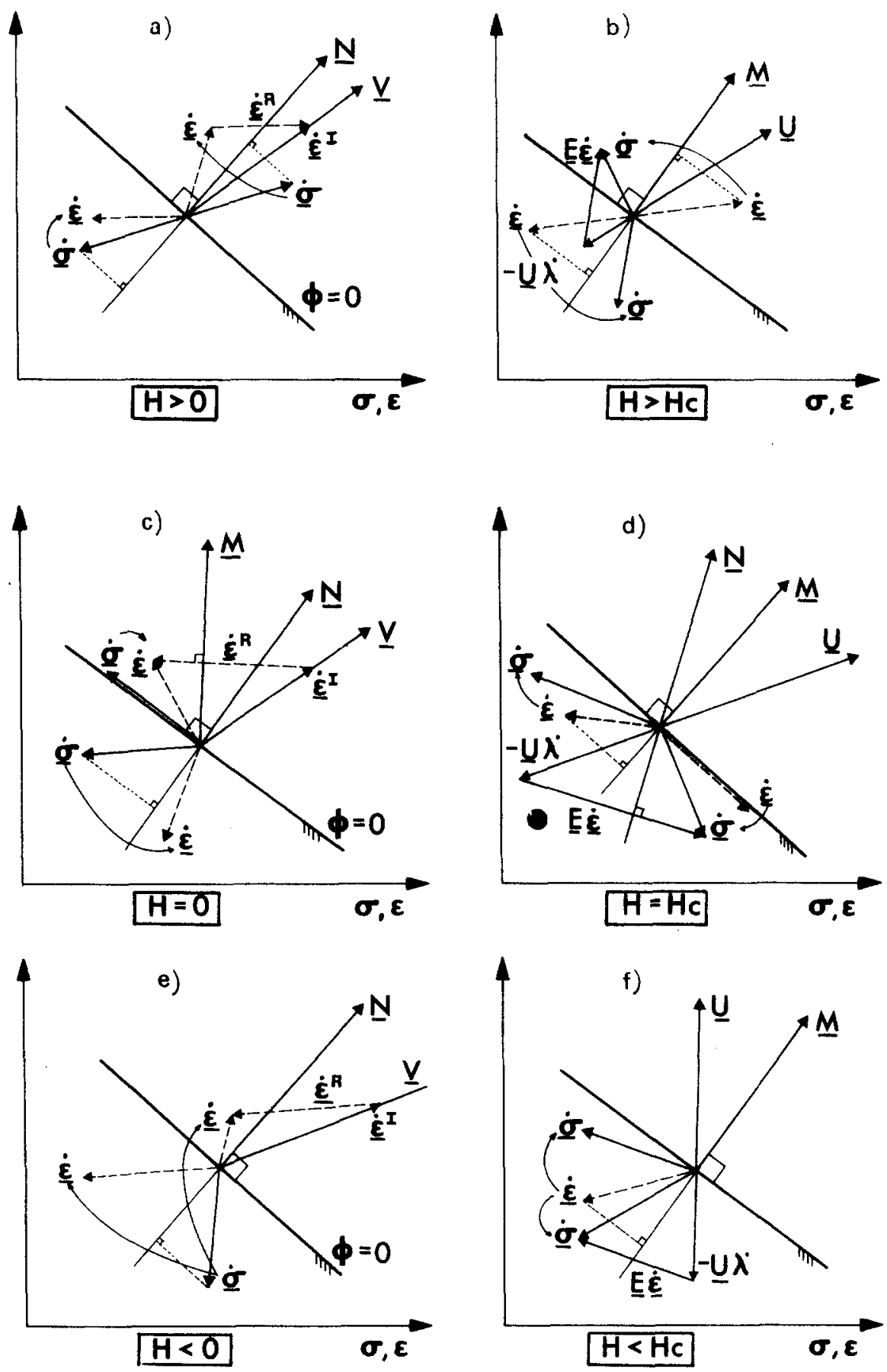

Fig. 5. Schematic representation of rate constitutive laws with elastoplastic coupling or/and nonassociated flow rules for various ranges of hardening moduli: direct laws on the left; inverse laws on the right.

as stable at a current state $\left(\sigma_{i j}, \Pi\right)$ if and only if $W \geq 0$ for all $\dot{\epsilon}_{i j}$. Due to positive definiteness of $E_{i j k l}, W>0$ for any purely elastic incremental process $(\dot{\lambda}=0)$. Therefore, only the set of yielding $(\dot{\lambda}>0)$ processes need to be examined. Since only the sign of $W$ matters for stability, one can assume $\dot{\lambda}=1$ and, hence, $\dot{\phi}=0$.

Thus, by substituting (2.11) into (4.1), the stability condition (4.1) is equivalent to the requirement:

$$
\min _{\dot{\epsilon}_{i j}} \frac{1}{2}\left\{\dot{\epsilon}_{i j} E_{i j k l} \dot{\epsilon}_{k l}-\dot{\epsilon}_{i j} E_{i j k l} V_{k l}\right\}
$$

subject to:

$$
M_{i j} \dot{\epsilon}_{i j}=H-H_{c}
$$

The optimal vector $\dot{\epsilon}_{i j}^{0}$ and the optimal value $W_{0}$ for the above equality-constrained, quadratic, strictly convex minimization, turn out to be (see Appendix):

$$
\begin{gathered}
\dot{\epsilon}_{i j}^{0}=\frac{1}{2} V_{i j}-\omega N_{i j} \\
2 W_{0}=\omega^{2} N_{i j} E_{i j k l} N_{k l}-\frac{1}{4} V_{i j} E_{i j k l} V_{k l}
\end{gathered}
$$

having set:

$$
\omega=\left(\frac{1}{2} H_{c}-H\right)\left(N_{i j} E_{i j k l} N_{k l}\right)^{-1}
$$

Before examining the above results, it is worth noting that in the case of perfectly plastic behaviour, $H=0$, coupling and/or lack of normality necessarily generate material instability in the sense of criterion (4.1). 


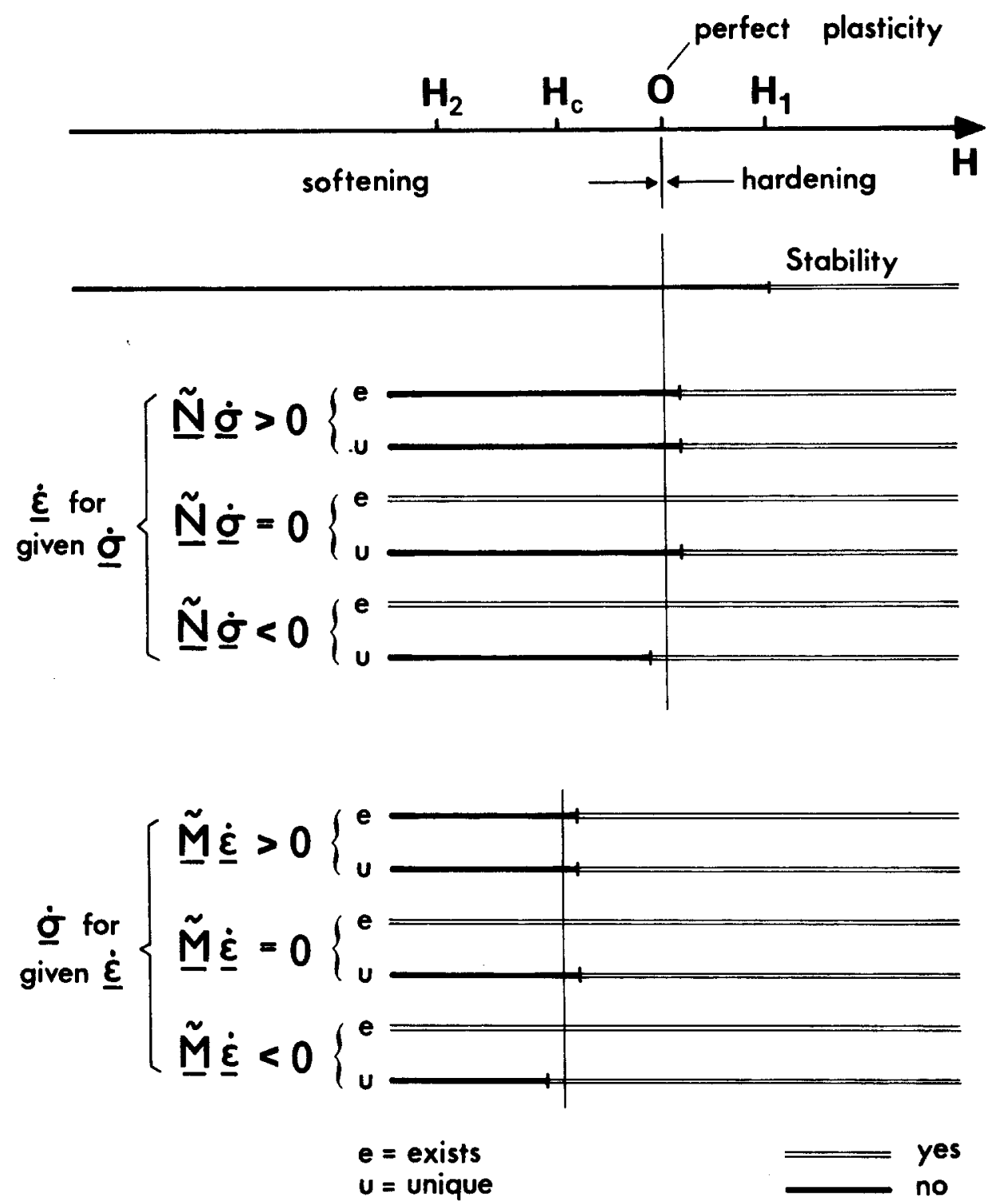

Fig. 6. Existence, uniqueness of rate response and stability for various ranges of the hardening modulus.

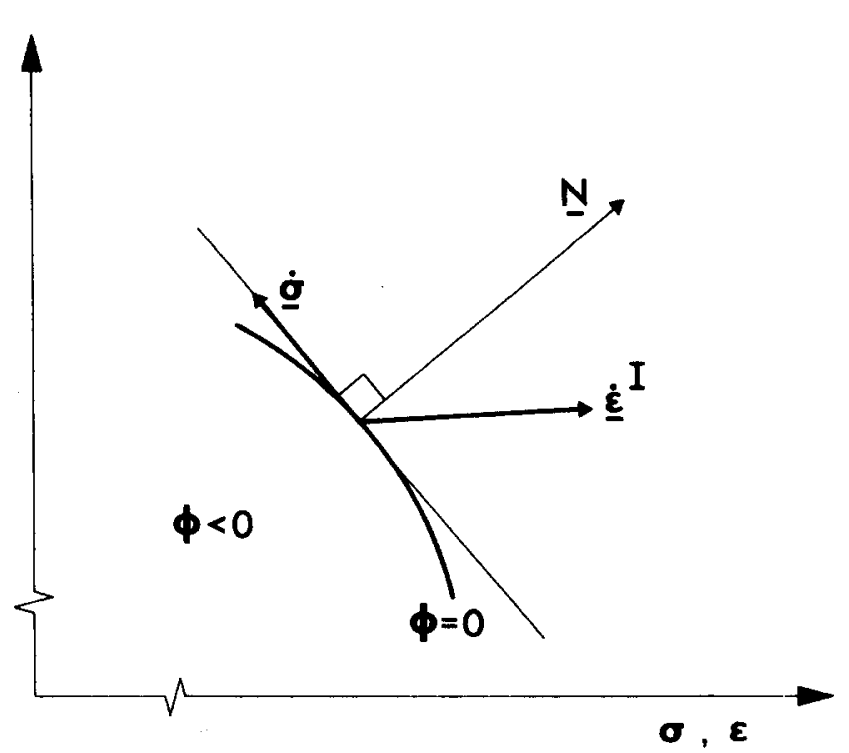

Fig. 7. Illustration of material instability due to coupling or deviation from normality in non-hardening situations. namely $W<0$ for some strain rates. This can be shown either through (4.5)-(4.6) or, simply, by referring to the special stress path of Fig. 7. In fact, for this path the irreversible, non-recoverable part of the work is $2 W^{I} \equiv \dot{\epsilon}_{i j} \dot{\epsilon}_{i j}^{I}<0$; reducing the norm $\left\|\dot{\sigma}_{i j}\right\|$, the recoverable part $2 W^{R} \equiv \dot{\sigma}_{i j} \dot{\epsilon}_{i j}^{R}=\dot{\sigma}_{i j} \quad E_{i j k l} \quad \dot{\sigma}_{k l}>0$ decreases quadratically and may be made smaller than $W^{I}$, since $\dot{\sigma}_{i j}^{I}$ is independent from the entity of $\dot{\sigma}_{i j}$.

Clearly, hardening $(H>0)$ can compensate for the destabilizing effects of coupling and/or non-normality. It is of interest to know what is the minimum hardening modulus $H$ which restores stability despite these effects.

Setting $W_{0}=0$ in equation (4.5) account taken of (4.6) and solving the second order equation for $H$, one obtains:

$$
2 H_{1,2}=H_{c} \pm\left(V_{i j} E_{i j k l} V_{k l} \cdot N_{r s} E_{r s m n} N_{m n}\right)^{1 / 2}
$$

Simple algebraic manipulations on equations (4.5) and 


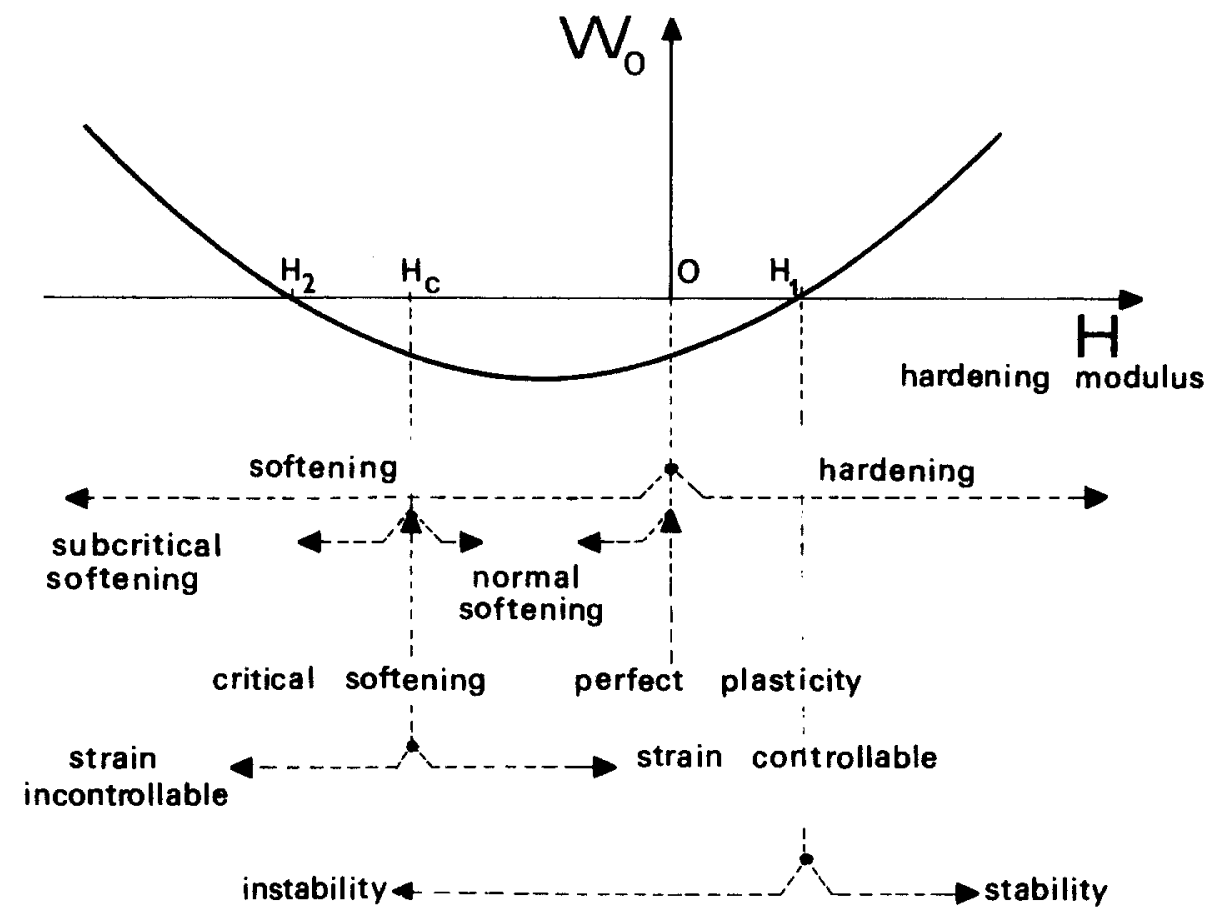

Fig. 8. Ranges of hardening modulus and their mechanical characteristics. $W_{0}$ represents the minimum second order work density over all admissible strain increments under the condition $\dot{\lambda}=1$.

(4.6), as outlined in Appendix, lead to the following conclusions (see $W_{0}$ vs $H$ plot in Fig. 8):

$$
\begin{gathered}
W_{0}<0 \text { for } H_{2}<H<H_{1} \\
W_{0} \geq 0 \text { for } H \geq H_{1} \text { and for } H \leq H_{2}
\end{gathered}
$$

It is shown below that:

$$
H_{1}=\frac{1}{2} H_{c}+\frac{1}{2}\left(V_{i j} E_{i j k l} V_{k l} \cdot N_{r s} E_{r s m n} N_{m n}\right)^{1 / 2}>0 .
$$

if

$$
V_{i j} \neq N_{i j} \chi
$$

$\chi$ being any arbitrary positive number.

In fact, through suitable re-definition of the yield function $\phi$ and, hence, of its gradient $N_{i j}$, it is always possible to have $N_{i j} E_{i j k l} N_{k l}=V_{i j} E_{i j k l} V_{k l}$. Let $q$ be the common value of these expressions. Since $E_{i j k l}$ is positive definite, for any constant $q, \epsilon_{i j} E_{i j k l} \epsilon_{k l}=q$ represents an ellipsoid in the 6-dimensional space of the independent components of the (symmetric) tensor $\dot{\epsilon}_{i j}$. The strict convexity of this hyper-surface allows one to write:

$$
N_{i j} E_{i j k l}\left(N_{k l}-V_{k l}\right)>0
$$

since $N_{i j}$ and $V_{i j}$ represent two points on the surface and $E_{i j k l} N_{k l}$ is the gradient (outward normal) vector in the former point. From equation (4.11) the circumstance stated in (4.10) follows, account taken of (2.14). Clearly, $H_{1}=0$ if and only if $V_{i j}=N_{i j} \chi$.

On the basis of inequality (4.11), it is also readily seen that:

$$
H_{2}=\frac{1}{2} H_{c}-\frac{1}{2}\left(V_{i j} E_{i j k l} V_{k l} \cdot N_{r s} E_{r s m n} N_{m n}\right)^{1 / 2}<H_{c}
$$

if

$$
V_{i j} \neq N_{i j} \chi
$$

whereas $H_{2}=H_{c}$ if and only if $V_{i j}=N_{i j} \chi$.

Over the range $\mathrm{H} \leq \mathrm{H}_{2}$, the second-order work was found to be nonnegative according to (4.9). However, because of inequality (4.12), this fact does not imply stability; in fact, the whole range $H \leq H_{c}$ is characterized by non existence of infinitesimal stress response for a set of infinitesimal strain increments (see Section 3), i.e. by a discontinuity ('jump') in the stress response. In other terms, there are infinitesimal strain increments such that the corresponding stress increments are finite (and, hence, not susceptible to be represented by the rate flow rules) and the work done is negative of the $1^{\text {st }}$ order (and, hence, not expressed in form (4.1)). Therefore the value $H_{c}$ can be referred to as 'perfectly brittle behaviour' modulus or 'critical softening' modulus, the range $H \leq H_{c}$ as the 'subcritical softening' range for the hardening modulus $H$ of the material behaviour [18]. As a conclusion, the material stability in the sense of criterion (4.1) is guaranteed if and only if:

$$
\begin{aligned}
H \geq \frac{1}{2}\left[V_{i j} E_{i j k l} V_{k l} \cdot N_{r s} E_{r s m n} N_{m n}\right)^{1 / 2} & \left.-N_{i j} E_{i j k l} V_{k l}\right]=H_{1}
\end{aligned}
$$

For a given deviation from normality of the plastic strain rate vector and given coupling, the r.h. side of inequality (4.13) provides the hardening modulus $H_{1}$, which represents the minimum hardening required for stability in the sense of (4.1), in the presence of nonassociative plastic flow law and coupling. It is worth stressing that, according to equation (4.10), $H_{1}=0$ if and only if the irreversible strain rate vector which represents 
the tensor $\dot{\epsilon}_{i j}^{I}$, is directed as the outward normal to the current yield surface $\left(V_{i j}=\chi N_{i j}\right)$. This occurs either when the plastic flow rule is associated $\left(\dot{\epsilon}_{j}=\lambda N_{i j}\right)$ and no coupling exists $\left(\dot{\epsilon}_{i j}^{c}=0\right)$, or when, exceptionally, the friction-induced deviation from normality of $\dot{\epsilon}_{j}$ is compensated for by the effects $\dot{\epsilon}_{i j}^{c}$.

The above conclusions on material stability are summarized in Fig. 6 and 8. In Fig. 6 they are contrasted with those of the preceding Section. It is worth noting, as visualized in Fig. 6, that in the hardening modulus range $0<H<H_{1}$, material instability in the sense of criterion (4.1) is accompanied by existence and uniqueness of the rate response. In other terms, in this range instability meant as negative second-order work, is exhibited by an incremental behaviour characterized by a one-to-one correspondence between stress rate tensors and strain rate tensors. This peculiarity of the nonassociative flow laws of elastoplasticity (which disappears in associative laws where $H_{1}=0$ ) seems to have been overlooked so far.

It is worth noting that, in a typical mechanical system made of stable materials and susceptible of instability due to geometric effects (such as a perfectly straight elastic-plastic column or Shanley's model of it), as the load increases, loss of incremental uniqueness ('bifurcation') is encountered first and the instability onset later $[26,27]$. If the decreasing hardening modulus is regarded here as a destabilizing effect (parallel to the load on the column), the above sequence, which is normal in structural mechanics, is found here to occur in the reverse order for nonassociative flow rules in multiaxial cases.

In the next two sections the meanings and implications of the characteristic values $H_{1}, H_{2}$ and $H_{c}$ of the hardening modulus are further investigated.

\section{SPECIALIZATION TO STANDARD COMPRESSION TESTS}

In order to clarify the mechanical meaning of some aspects of the present theory, the usual compression test ('confined' or 'unconfined', i.e. in the presence or in the absence of fluid-generated lateral pressure) will be discussed below and interpreted on the basis of the preceding results. The stress path corresponding to a customary 'unconfined' compression is indicated in Fig. 1 by the dotted straight line a cursive through the origin. It can be noticed in this figure, that the material model assumed there implies softening behaviour $(H<0)$ at the onset of yielding in the uniaxial test. For 'confined' compression tests, i.e. for tests performed in the presence of constant lateral pressure, hardening behaviour may show up, as for path c cursive in Fig. 1.

Let all components of $\sigma_{i j}$ vanish except the first one, which becomes the only nonzero principal stress rate $\dot{\sigma}_{1}$ (along the axis 1 of the specimen). Equation (3.2), account taken of (3.3), supplies the slope of the uniaxial stress state plot (see Fig. 9) in the form:

$$
\mu=H E_{1}\left(H+N_{1} E_{1} V_{1}\right)^{-1}
$$

where $E_{1}$ denotes the Young modulus in the direction of axis 1 ( $E$ for isotropic materials), $N_{1}$ and $V_{1}$ indicate the corresponding components of the vectors $\mathbf{N}_{i j}$ and $V_{i j}$.

Equation (5.1) shows that $H>0$ implies $\mu>0$, $H=0$ implies $\mu=0$; namely, hardening (e.g. path c in Fig. 1) and perfectly plastic behaviour (e.g. path b) in the six-dimensional stress space, would result in a sloping-up and horizontal branch, respectively, in Fig. 9. Through equation (5.1), one observes that for

$$
H_{c}^{(1)}=-\dot{N}_{1} E_{1} V_{1}
$$

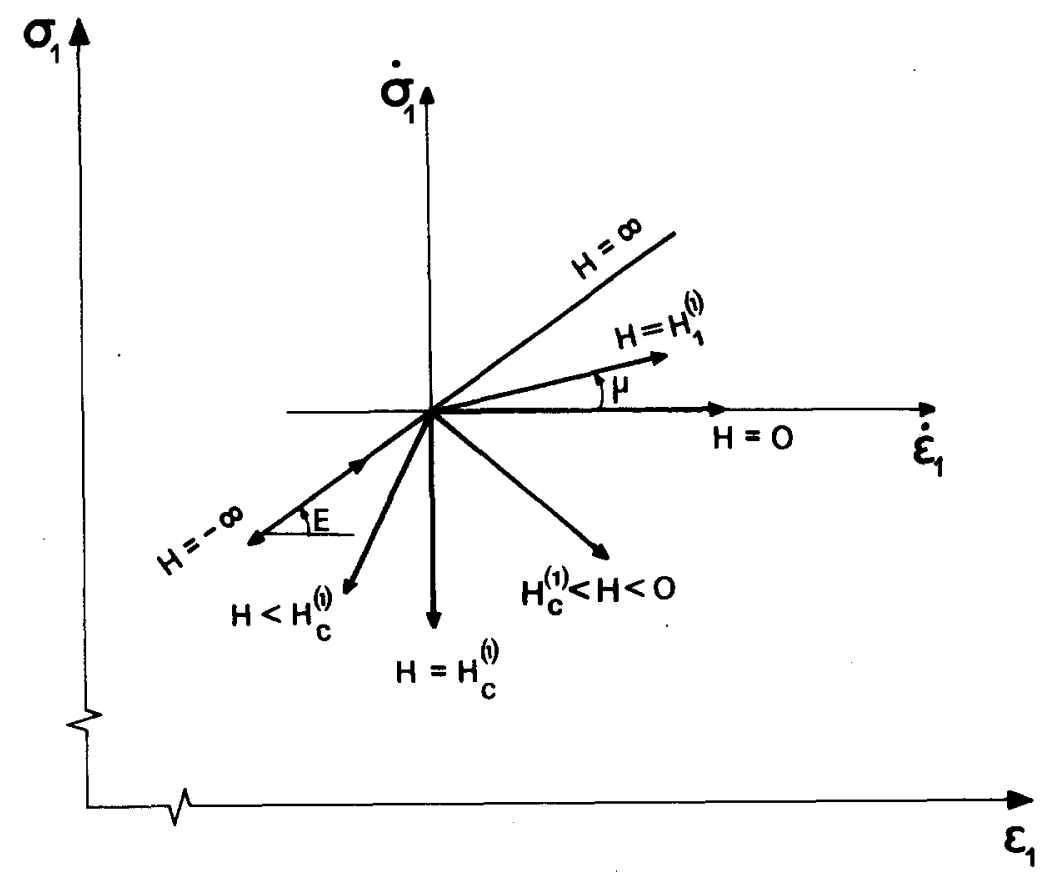

Fig. 9. Ranges of hardening modulus for one-dimensional systems such as compression specimens. 
$\mu=-\infty$, i.e. the uniaxial plot falls down vertically, i.e. the stress $\sigma_{1}$ as a function of $\epsilon_{1}$ exhibits a discontinuity, in the sense that there is an infinity of $\sigma_{1}$ for the same $\epsilon_{1}$. This situation, which can be referred to as critical softening, separates two ranges of softening behaviour $(H<0)$, characterized by the following mechanical features (see Fig. 9).

(a) $H>H_{\mathrm{c}}^{(1)}$ ('normal' softening in compression tests): the specimen is unstable in the sense of Section 4 ; the test can be performed as a quasi-static process only by controlling the strain $\epsilon_{1}$ (through a stiff loading device), not by controlling the stress $\sigma_{1}$.

(b) $H<H_{c}^{(1)}$ ('subcritical' softening in compression tests): the second-order work is positive both in the elastic unloading and in the yielding path; however, for $\delta \epsilon>0$ a finite decrease ('a jump') in stress occurs, so that the work related to the stress and strain changes is of the first order and negative; the specimen exhibits a strong 'intrinsic' instability, since it cannot be kept under control as a quasi-static system, even with a perfectly rigid testing machine.

It is worth noting that of concern here is merely the description of the main overall, phenomenological aspects of material behaviour in the framework of some simple kind of constitutive law. The above aspects, however, are connected with a variety of complex phenomena within the specimen and at the microscale.

For rocks, an analysis of these phenomena can be found in ref $[10,11]$, where normal and subcritical softening in compression tests are discussed on the basis of abundant experimental results, under the denomination of 'I class' and 'II class' of failure behaviours, respectively. The I class was studied e.g. in some marble (Fig. 10a), the II (or 'self-sustained fracture') was observed e.g. in some basalt (Fig. 10b).

Similar behaviour can be reproduced by structural trusslike models composed of elastoplastic constituents, allowing for geometric effects on equilibrium [27].
Whatever the complexity of the phenomena occurring inside of it, a specimen in the compression test represents a system acted upon by external agencies through only one degree of freedom. The critical softening threshold $H_{c}^{(1)}$, equation (5.2), for the compression test corresponds to a discontinuity of the stress response in a system of the above type; this, however, is stress-constrained laterally, in the sense that $\sigma_{2}$ and $\sigma_{3}$ are fixed and, hence, stress changes may occur only in terms of $\sigma_{1}$. It is worth noting that, in general

$$
H_{c}^{(1)} \neq H_{c}
$$

In fact, the critical hardening modulus value $H_{c}$, by its very definition Section 3, corresponds to a discontinuity in the stress response of a material specimen which is conceived (in contrast to the afore-mentioned situation) as acted upon by the external agencies through all its degrees of freedom, i.e. all independent strain components, so that there are no stress constraints. It can be conjectured that in most cases $H_{c}^{(1)} \geq H_{c}$, but this point and its mechanical interpretation should be pursued elsewhere.

As for the stability criterion (4.1), resting on the notion of virtual infinitesimal geometry disturbances, it clearly applies unaltered (i.e. for any $\dot{\epsilon}_{i j}$ ) to a hypothetical experimental situation where stresses are prescribed; therefore, a stress-controlled compression test implies no change of the characteristic values $H_{1}$ and $\mathrm{H}_{2}$, equation (4.7), which define hardening modulus ranges of positive second order work for material.

For an ideal, perfectly rigid testing machine, i.e. such that the platens represent imposed kinematic boundary conditions, the minimization (4.2) of the second order work should be performed under the additional constraints $\dot{\epsilon}_{1}=0$.

For a loading device with a finite elastic stiffness $k$, the work $W$ of equation (4.1) should be supplemented by an addend proportional to $k \dot{\epsilon}_{1}^{2}$ and pertaining to

\section{a) b)}
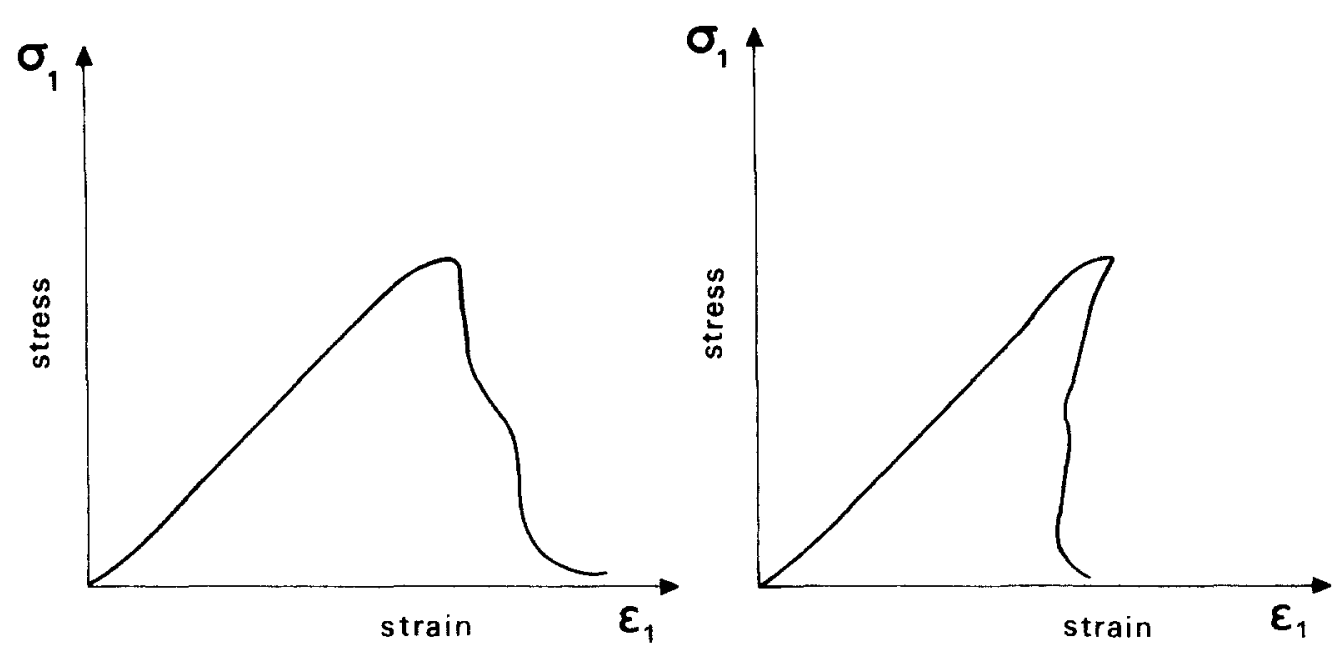

Fig. 10. Classes of the softening behaviour (from Ref. [10]): (a) normal softening or Class I: (b) subcritical softening or Class II. 
the machine, before the optimization $(4.2)-(4.3)$ be carried out.

In both the above cases the hardening moduli $H_{1}^{(1)}$, $H_{2}^{(1)}$ obtained as above indicated, will be different from $\mathrm{H}_{1}$ and $\mathrm{H}_{2}$ obtained in Section 4. In fact, the former parameters, in contrast to the latter ones, are characteristics of the system formed by the specimen and the testing machine, rather than of the material alone.

A characteristic value $H_{1}^{(1)}$ thus calculated (maximum of the instability interval of hardening moduli), may be positive if $H_{1}$ for the general case, equation (5.1), is so due to the deviation of $V_{i j}$ from $N_{i j}$ (i.e. because of coupling and/or frictional effects). Also the slope $\mu_{1}$ corresponding to $H_{1}^{(1)}>0$ through (5.1) will be positive. In this case in the $\sigma_{1}$ vs $\epsilon_{1}$ relationship for a compression text, there will be a range of positive slopes $0<\mu \leq \mu_{1}$ corresponding to unstable situation in the sense of criterion (4.1).

The physical consistency of this rather unexpected circumstance becomes clear, if one notes that geometric disturbances apt to check stability involve second order work addends $\frac{1}{2}\left(\dot{\sigma}_{2} \dot{\epsilon}_{2}+\dot{\sigma}_{3} \dot{\epsilon}_{3}\right)$ due to transversal strain increments, besides $\frac{1}{2} \dot{\sigma}_{1} \dot{\epsilon}_{1}$ in the direction of the specimen axis.

The above remarks on material instability for positive hardening bear a resemblance to the experimental observation that self-sustained crack propagations preceded the peaks of the stress-strain curves in uniaxial tests on hard rock specimens[28].

\section{LOCI OF STABLE INCREMENTAL STRAIN AND STRESS PATHS}

Consider again the incremental behaviour as it would appear in an ideal experiment in which all strain components can be separately controlled. At a situation in which the material exhibits instability in the sense of the criterion (4.1), i.e. with a hardening modulus $H$ internal to the interval $H_{1}-H_{2}$, equation (4.7), there exists a set of incremental yielding paths along which the second order work is negative. The work in equation (4.1), for yielding paths, can be expressed as a quadratic form using either Eq. (3.5) or (3.2)

$$
2 W=\dot{\epsilon}_{i j} B_{i j k l} \dot{\epsilon}_{k l}=\dot{\sigma}_{i j} A_{i j k l} \dot{\sigma}_{k l}
$$

The locus $A$ of unstable strain rates will be studied below. For the sake of simple geometrical visualisation, the following discussion will be referred to isotropic materials in the three-dimensional space of the principal strains, Fig. 11, i.e. in terms of three components vectors represented by underlined symbols. Therefore, it will be set henceforth $i=j$ and $k=l$, and single indeces $i$ and $k$ will refer to the principal directions. However, all conclusions hold unaltered for six strain components, required for the description of general anisotropic material behaviour.

Setting $W=0$ in equation (6.1), taken of equation
(3.3), we obtain in the $\dot{\epsilon}_{i}$ space the quadratic surface:

$$
\dot{\epsilon}_{i}\left(E_{i k}-\frac{1}{H-H_{c}} U_{i} M_{k}\right) \dot{\epsilon}_{k}=0
$$

This equation represents a cone $\Gamma$ with the vertex in the origin of the $\dot{\epsilon}_{i}$ space and forms the boundary of the locus $A^{\prime}$ of all $\dot{\epsilon}_{i}$ such that $W \leq 0$. The instability locus will be the intersection between $A^{\prime}$ and the locus $A^{\prime \prime}$ of all admissible and yielding $\dot{\epsilon}_{i}$ for the given situation $\Sigma$. The locus $A^{\prime \prime}$ is represented by a half-space or by a plane, depending on the hardening modulus range, precisely (see Section 3):

$$
\begin{gathered}
M_{i} \dot{\epsilon}_{i i}>0, \text { for } H>H_{c} ; M_{i} \dot{\epsilon}_{i}=0, \text { for } H=H_{c} ; \\
M_{i} \dot{\epsilon}_{i}<0, \text { for } H<H_{c}
\end{gathered}
$$

In order to examine the properties of the locus $A^{\prime}$, it is convenient to refer to a new coordinate system $\eta_{i}$ :

$$
\eta_{i}=E_{i k}^{\prime} \dot{\epsilon}_{k}
$$

where $\left[E_{i k}^{\prime}\right]$ represents the (positive definite, symmetric) square root matrix of $\left[E_{i j}\right]$, and can be expressed in the following form [29]:

$$
E_{i k}^{\prime}=\lambda_{h}^{1 / 2} t_{i h} t_{h k}
$$

$\lambda_{h}$ being the $h$-th eigenvalue of $\left[E_{i k}\right], t_{i h}$ the $i$-th component of the $h$-th normalized eigenvector. It can be realized from equation (6.5) that $\left[E_{i h}^{\prime}\right]\left[E_{h l}^{\prime}\right]=\left[E_{i l}\right]$. Let us set:

$$
\begin{gathered}
N_{i}^{\prime}=E_{i k}^{\prime} N_{k}, V_{i}^{\prime}=E_{i k}^{\prime} V_{k} \\
n_{i}=N_{i}^{\prime}\left(N_{k}^{\prime} N_{k}\right)^{-1 / 2}, v_{i}=V_{i}^{\prime}\left(V_{k}^{\prime} V_{k}^{\prime}\right)^{-1 / 2}
\end{gathered}
$$

Making use of the unit vectors (6.7), equation (6.2) becomes:

$$
\eta_{i} v_{i} n_{k} \eta_{k}=C \eta_{i} \eta_{i}
$$

where, through (6.2):

$$
\begin{aligned}
C=\left(N_{i}^{\prime} N_{i}^{\prime}\right)^{-1 / 2}\left(V_{k}^{\prime} V_{k}^{\prime}\right)^{-1 / 2}\left(H-H_{c}\right) & \\
= & \left(H_{1}-H_{2}\right)^{-1}\left(H-H_{c}\right)
\end{aligned}
$$

A directrix of the cone (6.8) can be conveniently singled out by intersecting it with the sphere of unit ray. This intersection curve is defined by the two equations:

$$
\begin{gathered}
\eta_{i} \eta_{i}=1 \\
\eta_{i} v_{i} n_{k} \eta_{k}=C
\end{gathered}
$$

Equation (6.11), obtained by accounting for equation (6.10) in (6.8), is easily seen to represent, in the $\eta_{i}$ space, a cylinder with generatrices orthogonal to the plane of $n_{i}$ and $v_{i}$. This plane intersects the cylinder in a hyperbola, the asymptotes of which are normal to vectors $n_{i}$ and $v_{i}$ (Fig. 11).

For $H>H_{c}$, (and, hence, $C>0$ ), the hyperbola intersects the straight lines along the vectors $n_{i}$ and $v_{i}$; its axis, and, hence, the coincident axis of the cone (6.8), are defined by the vector $n_{i}+v_{i}$ of the bisectrix of angle formed by $n_{i}$ and $v_{i}$ (Fig. 11a). All unstable paths $\eta_{\text {in }}$ (such that $W<0$ ) forming the instability locus $A$ are internal to this cone $A^{\prime}$ and directed inwards the half 

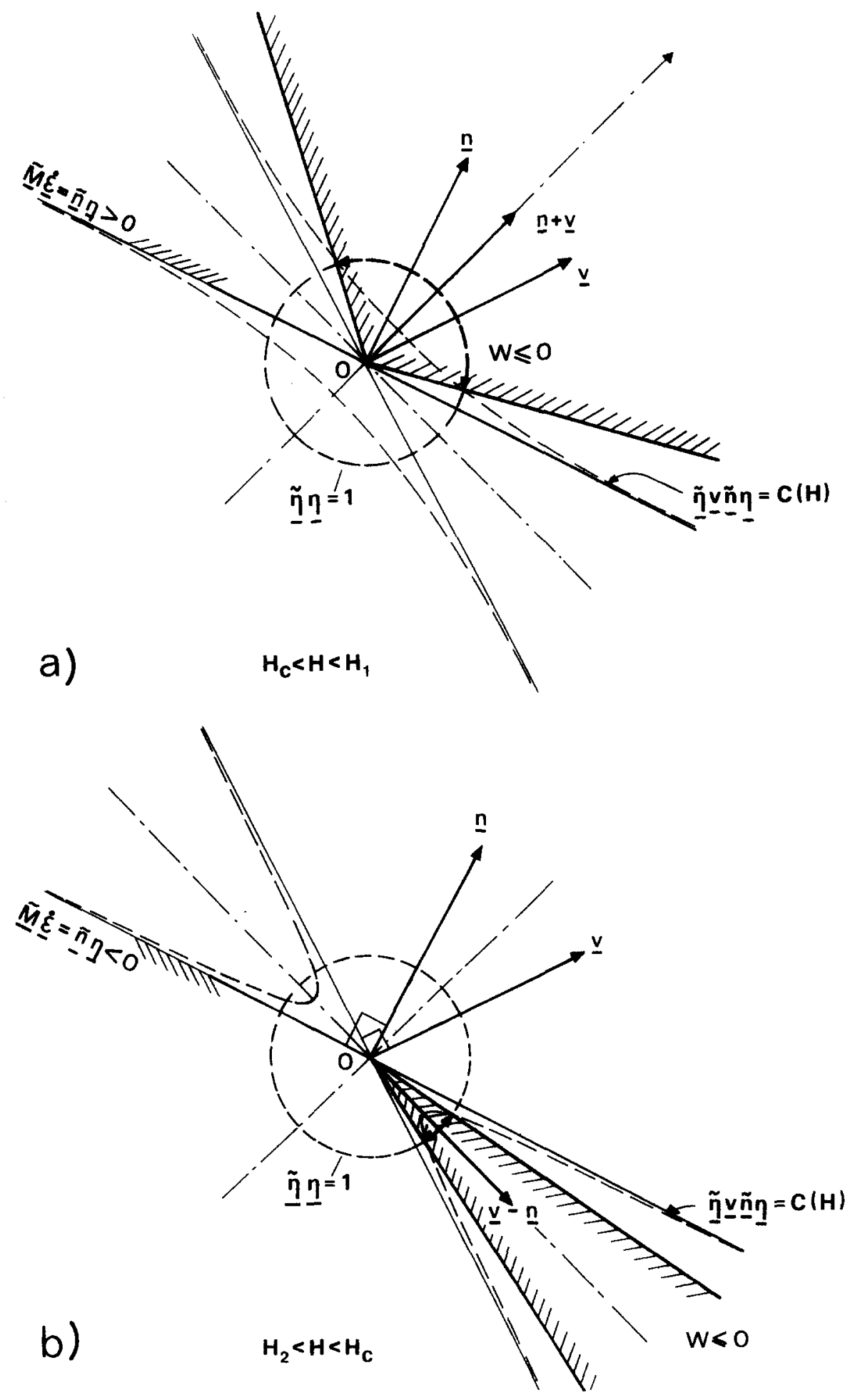

Fig. 11. Locus of unstable incremental strain paths for hardening moduli $H_{2}<H<H_{1}$, in the $\eta \equiv E^{1 / 2} \dot{\epsilon}$ space and in the $\hat{n} v$ plane: (a) for $H>H_{c}$; (b) for $H<H_{c}$ (subcritical softening).

space $A^{\prime \prime}\left(n_{i} \eta_{i} \geq 0\right)$ in order to comply with equation (6.3). The vector $n_{i}+v_{i}$ defines the weakest path for $H>H_{c}$, in the sense that it coincides with the direction in which the elastoplastic second order work $W$ attains its minimum over the set of all paths of the same length.

For $H<H_{c}$ (Fig. 11 b) the hyperbola does not intersect $n_{i}$ and $v_{i}$. Its (conjugate) axis and, hence, the coincident axis of the cone (6.8) are along vector $n_{i}-v_{i}$, which is normal to $n_{i}+v_{i}$ and contained in the plane of $n_{i}$ and $v_{i}$. The instability locus turns out to be as shown in Fig. $11 \mathrm{~b}$; the weakest path is defined by $v_{i}-n_{i}$.

For $H \rightarrow H_{1}$ the locus $A$ shrinks to its axis along $n_{i}+v_{i} ;$ for $H=H_{1}$ the only path with $W=0$ is $n_{i}+v_{i}$, while all other paths are strictly stable $(W>0)$. for $H>H_{1}$, the locus $A$ becomes imaginary.

For $H \rightarrow H_{c}$ the instability locus $A$ of Fig. 11a tends to coincide with the dihedral angle formed by the planes normal to $n_{i}$ and $v_{i}$ and containing them; for 
$H=H_{c}$, the locus reduces to the half of the plane $n_{i} \eta_{i}=M_{i} \dot{\epsilon}_{i}=0$, on which the orthogonality projection of $v_{i}$ lies.

All these geometrical remarks are easily justified by comparing the values $\chi_{s}$ and $\chi_{c}$ corresponding to the intersections of $\eta_{i}=\left(n_{i}+v_{i}\right) \chi$ with the sphere (6.10) and with the cylinders (6.11), respectively. Account taken of equations (2.14), (4.10) and (4.12) for $H_{c}, H_{1}$ and $\mathrm{H}_{2}$, respectively, it turns out that:

$$
\begin{aligned}
\chi_{s}^{2}= & \frac{1}{2}\left(H_{1}-H_{2}\right)\left(H_{1}-H_{2}-H_{c}\right)^{-1} ; \\
& \chi_{c}^{2}=C\left(H_{1}-H_{2}\right)^{2}\left(H_{1}-H_{2}-H_{c}\right)^{-2}
\end{aligned}
$$

For $\mathrm{H \rightarrow H} \mathrm{H}_{2} \leq \mathrm{H}_{\mathrm{c}}$ the locus $\mathrm{A}$ shrinks to its axis $v_{i}-\mathrm{n}_{i}$, whereas for $H \rightarrow H_{c}$ from below it expands to the whole dihedral angle formed by the planes normal to $n_{i}$ and $v_{i}$ and containing vector $v_{i}-n_{i}$ (Fig. 11b). These remarks can be readily justified in analogous way by considering intersections on $\eta_{i}=\left(v_{i}-n_{i}\right) \chi$.

The above geometrical conclusions can be transferred back to the strain space through the transformation equation (6.4). The vectors $N_{i}+V_{i}$ and $V_{i}-N_{i}$ corresponds to $n_{i}+v_{i}$ and $v_{i}-n_{i}$, respectively, through equation (6.4) and, therefore, they represent the weakest paths in terms of strain rates, for $H>H_{c}$ and for $H<H_{c}$, respectively. They are not the axes of instability loci, since the sphere becomes an ellipsoid through the transformation. However, all the remarks on the evolution of $A$ as $\mathrm{H}$ changes hold unaltered in the $\dot{\epsilon}_{i}$ space with reference to $N_{i}$ and $V_{i}$ instead of $n_{i}$ and $v_{i}$.

An analogous discussion in geometrical terms could be carried out in the principal stress rate space $\dot{\sigma}_{i}$; account taken of the noted analogy (Section 3) between direct and inverse laws.

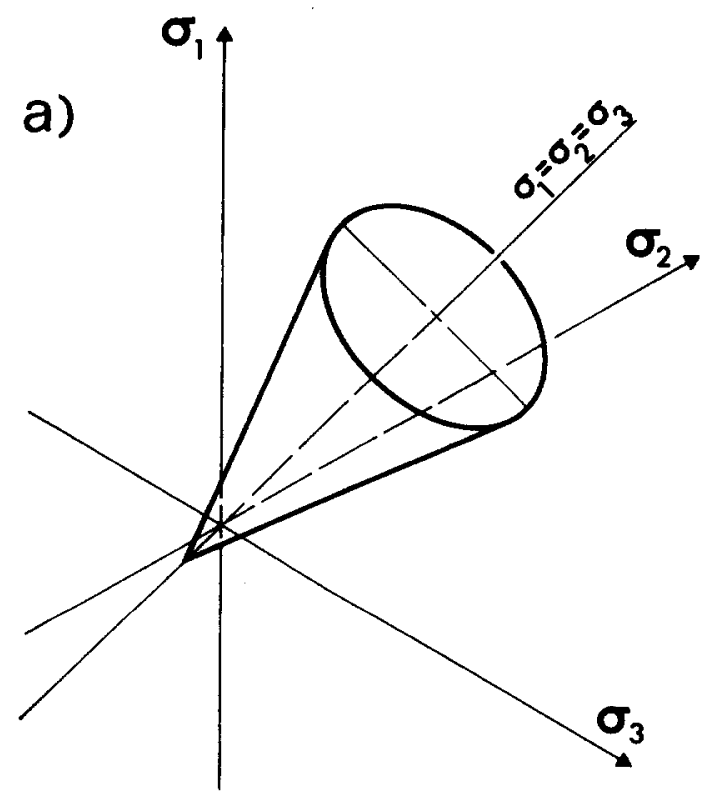

\section{APPLICATION TO PARTICULAR NONASSOCIATED LAWS}

Various expressions for the yield function $\phi$ and for the plastic potential $\psi$ were proposed in the literature in order to fit experimental data on geotechnical and rocklike media.

Three of these proposals concerning differentiable $\phi$ and $\psi$ (smooth yield and equipotential surfaces) formulated in terms of stress invariants alone (i.e. for isotropic materials) are considered below and the relevant characteristic values of hardening modulus are calculated on the basis of the present theory. The expressions (4.13) and (2.14) of the characteristic values $H_{1}$ and $H_{c}$, concerning stability in the sense of equation (4.1) and uniqueness of stress rate response to given strain rates (critical softening), respectively, are re-written here for cases where the elastic-plastic coupling is not accounted for i.e. nonnormality is regarded as a purely frictional or dilational effect [30].

$$
\begin{gathered}
H_{1}=\frac{1}{2}\left[-\frac{\partial \phi}{\partial \sigma_{i j}} E_{i j h k} \frac{\partial \psi}{\partial \sigma_{h k}}+\left(\frac{\partial \phi}{\partial \sigma_{i j}} E_{i j h k} \frac{\partial \phi}{\partial \sigma_{h k}}\right.\right. \\
\left.\left.\times \frac{\partial \psi}{\partial \sigma_{r s}} E_{r s m n} \frac{\partial \psi}{\partial \sigma_{m n}}\right)^{1 / 2}\right] \\
H_{c}=-\frac{\partial \phi}{\partial \sigma_{i j}} E_{i j h k} \frac{\partial \psi}{\partial \sigma_{h k}}
\end{gathered}
$$

The isotropic linear elastic tensor is:

$$
\mathrm{E}_{i j k l}=\frac{1}{3}(K-G) \delta_{i j} \delta_{k l}+\frac{1}{2} G\left(\delta_{i k} \delta_{j l}+\delta_{i l} \delta_{j k}\right)
$$

where $K$ and $G$ denote the bulk and shear modulus, respectively, and $\delta_{i j}$ is the Kronecker symbol.

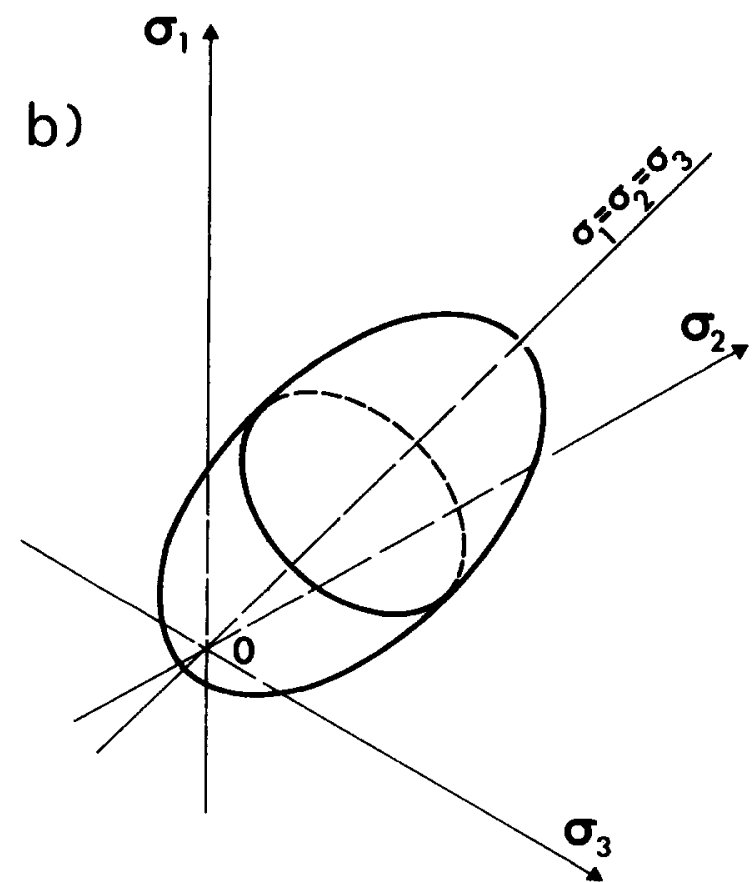

Fig. 12. Some typical yield conditions proposed for soils (a, Ref. [31]) and for rocklike media (b, Ref. [34]). 
(a) The classical Drucker-Prager yield condition for soils (a cone in the principal stress space, Fig. 12a) reads [31]:

$$
\phi=\alpha \mathbf{I}_{I}+\mathbf{I}_{\mathbf{I} \mathbf{l} d}^{1 / 2}-k=0
$$

where: $I_{I}=\sigma_{i i}$ is the first invariant of the stress tensor $\sigma_{i j} ; \mathrm{I}_{\text {IId }}$ the second invariant of its deviatoric part $s_{i j}=\sigma_{i j}-\frac{1}{3} \sigma_{k k} \delta_{i j} ; \alpha$ and $k$ are defined as follows:

$$
\begin{gathered}
3 \alpha^{2}=\sin ^{2} \rho\left(3+\sin ^{2} \rho\right)^{-1} \\
k^{2}=\left(3 c^{2}+\cos ^{2} \rho\right)\left(3+\sin ^{2} \rho\right)^{-1}
\end{gathered}
$$

as functions of the angle $\rho$ of internal friction and of the cohesion $c$. The parameter $c$ and possibly also $\rho$ can be made dependent on a measure of plastic deformations (e.g. plastic volumetric change), so that the hardening rule be specified (isotropic hardening in the case of variable cohesion and constant friction).

In order to accommodate in the constitutive law experimentally observed deviations from normality, the following plastic potential appears suitable in association with equation (7.4) [32]:

$$
\psi=\theta \alpha I_{I}+I_{1}^{1 / 2}
$$

where $\theta$ is another material parameter (nondimensional): usually $\theta<1$, since the dilatation is generally less than that implied by the normality rule (which holds for $(\theta=1)$.

On the basis of the above assumptions, the minimum hardening for stability is furnished by equation (7.1) in the form:

$$
H_{1}=\frac{G}{4}(\zeta \theta+1)\left\{\left[1+\zeta\left(\frac{1-\theta}{\zeta \theta+1}\right)^{2}\right]^{1 / 2}-1\right\}
$$

where: $\zeta=6 K / G \alpha^{2}$ and the critical softening modulus is supplied by equation (7.2) in the form:

$$
H_{c}=-\frac{G}{2}(\zeta \theta+1) .
$$

(b) The nonassociative constitutive law proposed by Jenike and Shield [33] combines the yield function (7.4) with the von Mises plastic potential, i.e. with equation (7.7) specialized to $\theta=0$. In the case $H_{c}=-\frac{1}{2} G$ from equation (7.9); $H_{1}$ is analogously given by equation (7.8) for $\theta=0$ and, e.g. attains the value $0.5184 G$ for $\rho=30^{\circ}$ and $G=2 K$.

(c) Ellipsoidal yield surfaces and plastic equipotential surfaces (Fig. 12b) were suggested in ref [8] and [34], namely expressions for $\phi$ and $\psi$ of the following type:

$$
\begin{gathered}
\phi=\left(\mathrm{I}_{I}-a_{1}\right)^{2}+b_{1} \mathrm{I}_{\mathrm{IId}}-d=0 \\
\psi=\left(\mathrm{I}_{I}-a_{2}\right)^{2}+b_{2} \mathrm{I}_{\mathrm{II} d}
\end{gathered}
$$

where $a_{1}, a_{2}, b_{1}, b_{2}$ and $d$ are material parameters, which generally depend on the plastic history (if $d$ alone does, isotropic hardening is described). On this basis one obtains

$$
\begin{aligned}
& H_{1}=G b_{1} b_{2} \mathrm{I}_{\mathrm{IId}}\left(\xi \frac{\mathrm{I}_{1}-a_{2}}{\mathrm{I}_{1}-a_{1}}+1\right) \\
& \times\left\{\left[1+\xi \frac{b_{2}}{b_{1}}\left(\frac{a_{2}-a_{1}\left(b_{1} / b_{2}\right)}{\xi\left(\mathrm{I}_{1}-a_{2}\right)+\mathrm{I}_{1}-a_{1}}\right)^{2}\right]^{1 / 2}-1\right\}
\end{aligned}
$$

where

$$
\xi=6 \frac{K}{G} \frac{\left(I_{1}-a_{1}\right)^{2}}{I_{I I d} b_{1} b_{2}}
$$

and

$$
H_{c}=-2 b_{1} b_{2} \mathrm{I}_{\mathrm{IId}}\left(\xi \frac{\mathrm{I}_{1}-a_{2}}{\mathrm{I}_{1}-a_{1}}+1\right)
$$

\section{CONCLUSIONS}

In what precedes, incremental elastoplastic constitutive laws have been studied, with the hypothesis that the direction of the plastic strain rate vector (in the space stresses and strain superposed, where second order tensors are represented by vectors) does not depend on the stress rates. This hypothesis not 'assumes' but implies as consequences regular, 'smooth' points (no 'corners') in the yield and plastic-equipotential surfaces, and also rules out the so-called 'nonlinear' rate relations (like those contemplated in endochronic inelasticity, see, e.g. [35]).

The purposes were a systematic study and a mechanical interpretation of the special cases covered by the above kind of practically convenient laws, and an assessment of their aptitude to describe: (i) deviation from normality due to internal friction, (ii) coupling of elastic and plastic deformations and (iii) various aspects of softening behaviour. All these phenomena are of engineering interest for geotechnical media, particularly rocks and rock masses, and probably for other rock-like media (such as concrete and some materials used in nuclear [34] and chemical engineering).

The results achieved can be summarized as follows:

(a) The dependence of elastic moduli on plastic deformations merely requires a generalization of the notion of the 'irreversible' strain rates, but can be directly accommodated in incremental nonassociated flow rules of elastoplasticity.

(b) The deviation from normality of the irreversible strain rate tensor implies that there is a range of positive hardening moduli $\left(0<H<H_{1}\right)$ for which the material is unstable according to the criterion of positive second order work; nevertheless, a one-to-one correspondence between stress rate and strain rate vectors still holds within this range. Therefore, ignoring elasticplastic coupling and internal fraction, and consequent lack of normality, makes the analysis unsafe, in view of their above unstabilizing effects. On the other hand, local instantaneous unstable behaviour can be compensated for by stable behaviour of surrounding zones, so that the overall behaviour of rock masses can be still stable [23]. 
(c) When stability in the sense of positive secondorder work does not hold, for a hardening modulus range $H_{2}<0<H_{1}$, a cone defines the locus of the incremental strain paths for which the second-order work is negative, while it is positive for all the external paths.

(d) The characteristic hardening modulus $H_{c}$, contained in the above range $\mathrm{H}_{1}-\mathrm{H}_{2}$, defines 'perfectly brittle' ('critical' softening) behaviour in a multidimensional system, and provides the demarkation between normal softening or 'class I' and subcritical softening or class II behaviours (the latter being characterized by 'stress jumps' in kinematically controlled specimens or systems).

(e) The above characteristic hardening moduli $\mathrm{H}_{1}, \mathrm{H}_{2}$, $H_{c}$ are not the same as those which can be obtained from the same point of view and same approach, when (as in compression confined or nonconfined tests) some stress component is controlled by the surrounding environment, whereas the corresponding strain component is free as a consequence.

Acknowledgements - One of the authors, T. Hueckel, acknowledges with gratitude the support of the Exchange Programme between the National Research Council of Italy and the Polish Academy of Sciences during his stay at the Center for Computational Structura Mechanics, Department of Structural Engineering, Technical University (Politecnico) of Milan.

Received 10 January, 1978; in revised form 14 July, 1978

\section{REFERENCES}

1. Zienkiewicz O. C., Best B., Dullage C. \& Stagg K. G. Analysis of non linear problems in rock mechanics with particular reference to jointed rock systems, Proc. Belgrade Rock Mechanics II Congress Vol. 3, Paper 8-14 (1970).

2. Goodman R. E., Heuzé F. E. \& Bureau G. J. On modelling techniques for the study of tunnels in jointed rock, New Horizons in Rock Mechanics, Proc. 14th symp. on Roc. Mech., Penn. State Univ., June 1972, ASCE (1973).

3. Miller T. W. \& Cheatam J. B. A new yield condition and hardening rule for rocks Int. J. Rock Mech. Min. Sci. 9, 453-474 (1972)

4. Wroth P. \& Schofield A. Critical State Soil Mechanics, McGrawHill, London (1978).

5. Rice J. R. Inelastic constitutive relations for solids: an internal variable theory and its application to metal plasticity. J. Mech. Phys. Sol. 19, 433-455 (1971).

6. Drucker D. C. On the postulate of stability of material in mechanics of continua. J. Mécanique 3, 235-249 (1964).

7. Jaeger J. C. \& Cook N. G. W. Fundamentals of Rock Mechanics, Chapman and Hall, Science Paperbacks, New York (1971).

8. Roscoe K. H. \& Burland J. B. The generalized stress-strain behaviour of soft clays, Engineering Plasticity (Edited by Heyman J. and Leckie F.), pp. 535-610. Cambridge Univ. Press (1968)

9. Bieniawski Z. T. Deformational behaviour of fractured rock under multiaxial compression, Structure, Solid Mechanics and Engineering Design, Proc., Southampton, Part 1 (Edited by Te'eni), pp. 589-597 (1969).

10. Wawersik W. R. \& Fairhurst C. A study of brittle rock fracture in laboratory compression experiments. Int. J. Rock Mech. Min. Sci. 7, 561-575 (1970).

11. Hardy M. P., Hudson J. A. \& Fairhurst C. The failure of rock beams, Part I and II, Int. J. Rock Mech. Min. Sci. 10, 53-67 and $69-82$ (1973).

12. Rudnicki J. W. \& Rice J. R. Conditions for localization of deformation in pressure-sensitive dilatant materials. J. Mech. Phys. Solids, 25. $371-394$ (1975).

13. Bažant Z. P. Instability, ductility, and size effect in strain-softening concrete. Proc. ASCE, EM2, April, 331-344 (1976).
14. Maier G. On elastoplastic structures with associated stress-strain relations allowing for strainsoftening. Meccanica, 2, 55-63 (1967).

15. Dougill J. W. On stable progressively fracturing solids. $Z A M P$, 26, 423-437 (1976).

16. Nguyen Q. S. \& Radenkovic D. Stability of equilibrium in elastic plastic solids. Proc. IUTAM/IMU Symposium of Applications of Methods of Functional Analysis to Problems of Mechanics, Marseille (1975).

17. Di Maggio F. L. \& Sandler I. S. Material Model for Granular Soils. Proc. ASCE, EM2, June, pp. 935-950. (1971).

18. Maier G. Sui legami associati tra sforzi e deformazioni incrementali in elastoplasticitá. Rendiconti dell'Istituto Lombardo di Science e Lettere, A, 100, 809-830 (1966).

19. Mròz Z. On forms of constitutive laws for elastic-plastic solids. Archs Mech. 1, 18 (1966).

20. Maier G. Linears flow laws of elastoplasticity: a unified general approach. Rendiconti dell Accademia Naxionale dei Lincei, 142, A, 132-142 (1969).

21. Hueckel T. On plastic flow of granular and rocklike materials with variable elasticity moduli. Bull. Polish Acad. Sci., Ser. Sci. Tech., 23, 405-414 (1975).

22. Hueckel T. Coupling of elastic and plastic deformations of bulk solids. Meccanica-J. Ital. Ass. Theor. Appl. Mech. 11, 227-235 (1976)

23. Hueckel T. \& Maier G. Incremental boundary value problems in the presence of coupling of elastic and plastic deformations: a rock mechanics oriented theory. Int. J. Solids Structures 1 , 1-15 (1977)

24. Hill R. A general theory of uniqueness and stability in elasticplastic solids. J. Mech. Phys. Solids 6, 236-249 (1958).

25. Mandel J. Conditions de stabilité et postulat de Drucker. Rheology and Soil Mechanics, Proc. IUTAM Symp. Grenoble, 1964 (Edited by Kravtchenko J. and Sirieys P. M.) pp. 58-68. Springer, Berlin (1968)

26. Shanley F. R. The Column Paradox. J. Aero. Sci. 13, 678 (1946).

27. Maier G. \& Drucker D. C. Effects of geometry change on the essential features of inelastic behaviour. Proc. ASCE, EM4 819-834 (1973).

28. Bieniawski Z. T. Mechanism of brittle fracture of rock. Int. $J$. Rock Mech. Min. Sci. 395-406 (part I), 407-423 (part II) (1967).

29. Bellman R. Introduction to Matrix Analysis. McGraw, New York (1960).

30. Radenkovic D. Théorie des charges limites, extension à la mécanique des sols. Séminaire de Plasticité (Edited by Mandel J.), P.S.T., Min Air., No. 116, pp. 129-142 (1961).

31. Drucker D. C. \& Prager W. Soil mechanics and plastic analysis or limit design. Quart. Appl. Math. 10, 157-165 (1952)

32. Szczepinski W. Limit analysis and kinematics of granular media. P.W.N., Warsaw (1974). (in Polish).

33. Jenike A. W. \& Shield R. T. On the plastic flow of Coulomb solids beyond original failure. J. Appl. Mech. 27, 599-602 (1959).

34. Merkle J. G. An ellipsoidal yield function for materials that can both dilate and compact inelastically. Nucl. Eng. Design 12, 425-451 (1970).

35. Bažant Z. P. \& Bhat P. Endochronic theory of inelasticity and failure of concrete. Proc. ASCE, EM 3. June, pp. 701-722 (1976).

\section{APPENDIX}

Proof of some mathematical results in Section 4.

The equality-constrained minimization problem equations (4.2)-(4.3) reduces to the unconstrained minimization of the Lagrangean function

$$
\begin{aligned}
\mathrm{L}\left(\dot{\epsilon}_{i j}, \omega\right)=\frac{1}{2}\left(\dot{\epsilon}_{i j} E_{i j k l} \dot{\epsilon}_{k l}\right. & \left.-\dot{\epsilon}_{i j} E_{i j k l} V_{k l}\right) \\
& +\omega\left(M_{i j} \dot{\epsilon}_{i j}-H+H_{c}\right)
\end{aligned}
$$

where $\omega$ is a Lagrangean multiplier. The derivatives of $L$ with respect to all variables must vanish, i.e.:

$$
\begin{aligned}
& \frac{\partial L}{\partial \dot{\epsilon}_{i j}}=E_{i j k l} \dot{\epsilon}_{k l}-\frac{1}{2} E_{i j k l} V_{k l}+\omega M_{i j}=0 \\
& \frac{\partial L}{\partial \omega}=M_{i j} \dot{\epsilon}_{i j}-H+H_{c}=0
\end{aligned}
$$


The solution of these equations are equations (4.4) and (4.6). Substituting (4.4) and (4.6) into l.h.s. of (4.2), equation (4.5) is arrived at. The minimum work $W_{0}$ equation (4.5), may be written through (4.6) as follows:

$$
\begin{aligned}
2 W_{0}=\left[H^{2}\right. & -H H_{c}+\frac{1}{4}\left(H_{c}^{2}-V_{i j} E_{i j k l} V_{k l}\right. \\
& \left.\times N_{r s} E_{r s m n} N_{m n}\right] \cdot\left(N_{i j} E_{i j k l} N_{k l}\right)^{-1}
\end{aligned}
$$

The values $H_{1}$ and $H_{2}$ for which $W_{0}$ vanishes are given by equation (4.7). This polynomial can be re-written in terms of its roots $\mathrm{H}_{1}$ and $\mathrm{H}_{2}$, defined by equation (4.7):

$$
2 W_{0}=\left(H-H_{1}\right)\left(H-H_{2}\right)\left(N_{i j} E_{i j k l} N_{k l}\right)^{-1}
$$

From equation (4.7) one notices that:

$\left(H_{1}-H_{2}\right)=\left(V_{i j} E_{i j k l} V_{k l} \cdot N_{r s} E_{r s m n} N_{m n}\right)^{1 / 2}>0$

Since $N_{i j} E_{i j k l} N_{k l}>0$, it may be seen from equations (A-5) $(A-6)$ that the inequalities (4.8) and (4.9) are justified. 